\title{
Evolution of Public Transit Modes in a Commuter Corridor
}

Yanshuo Sun Ph.D.

Department of Civil and Environmental Engineering

University of Maryland, College Park

1173 Glenn Martin Hall, College Park MD 20742

yssun@umd.edu

Qianwen Guo Ph.D. (corresponding author)

Department of Finance and Investment

Sun Yat-sen Business School, Sun Yat-sen University

Bldg 388, 135 Xingangxi Rd, Guangzhou, China 510275

guoqw3@mail.sysu.edu.cn

Paul Schonfeld Ph.D.

Department of Civil and Environmental Engineering

University of Maryland, College Park

1173 Glenn Martin Hall, College Park MD 20742

pschon@umd.edu

Zhongfei Li Ph.D.

Department of Finance and Investment,

Sun Yat-sen Business School, Sun Yat-sen University

Bldg 388, 135 Xingangxi Rd, Guangzhou, China 510275

lnslzf@mail.sysu.edu.cn 


\begin{abstract}
This paper explores how the selection of public transit modes can be optimized over a planning horizon. This conceptual analysis sacrifices geographic detail in order to better highlight the relations among important factors. First, a set of static models is proposed to identify which type of service, e.g., bus only, rail only, or bus and rail, is the most cost-effective in terms of the average trip cost for given demand. After analyzing essential factors in a long-term planning process, e.g., economies of scale in rail extension and future cost discounting, a dynamic model incorporating such considerations is formulated to optimize the decision over a planning horizon. While analytical solutions can be obtained for some decision variables, the final model is solved with a graphical method by exploring the tradeoffs between the initial and recurring costs. Major findings from this study include: (a) there exists a minimum economic length for a rail line, which can be determined numerically; (b) economies of scale favor large extensions and excess supplied capacity; (c) the rail-only service is largely dominated by the feeder-trunk service, even in the long run.
\end{abstract}

Key Words: Public Transit Modes; Transportation System Evolution; Feeder Service; Rail Line Extension. 


\section{Introduction}

The type of public transit service provided should be determined mainly by the demand to be served, although other considerations, such as the desired level of service and budget constraint, can also play an important role in this decision process (Tirachini et al. 2010). Demand can change in its level, its distribution or both. On the supply side, the scale and type of service should be modified accordingly. Practitioners and researchers have long been interested in such a problem: how should the supplied service be adjusted in response to demand changes? Consider the evolution of public transit modes with demand in a commuter corridor as shown in Fig. 1. Assume that a single transit mode, e.g., bus transit, is used for serving commuters along a corridor. As the demand grows, service parameters of the bus transit, e.g., frequency and vehicle size, are modified to keep pace with the increase, while the technology remains the same. At some time, a new type of service which has a larger fixed cost but a considerably smaller marginal cost, e.g., rail transit, should be considered for partially or completely replacing the existing service. If the replacement is partial, new line structures might emerge, e.g., shared lines or lines with transfers (Jara-Díaz et al. 2012) from a single line. As the demand evolution continues, an additional transit mode might be introduced. In this paper, we first present a static method that identifies the threshold for introducing a new form of transit service and then develop a dynamic model for optimizing relevant parameters of the new service, such as the length ratio of rail to bus, over a planning horizon. Several terms, such as transit mode, form/type of transit service, are used interchangeably in this study. 


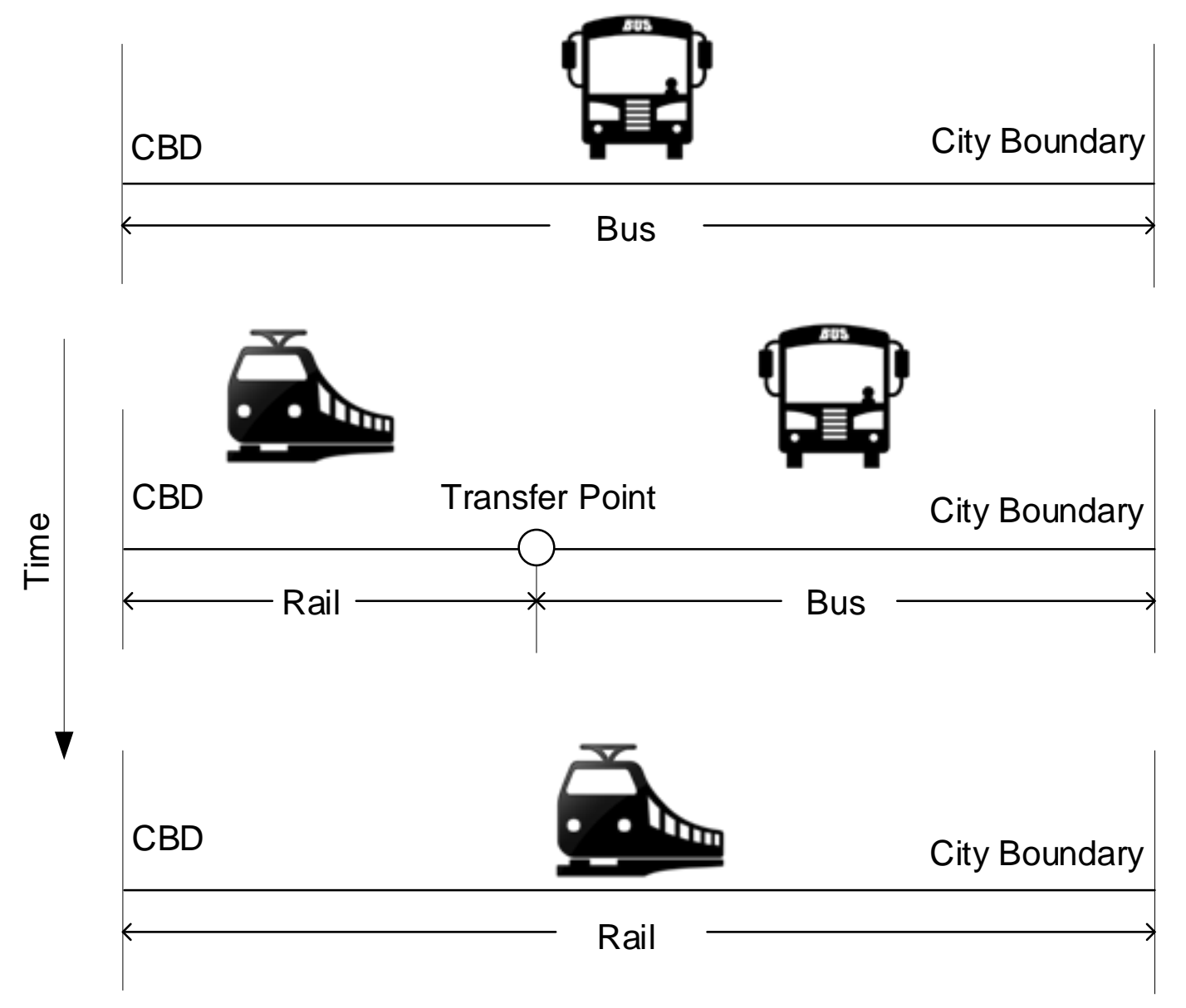

Fig. 1 Evolution of transit service types over time

The choice of transit mode has been studied for several decades since Vuchic's comparative analysis (Vuchic 1976) of alternative transit modes. Chang and Schonfeld (1991a) compared optimized fixed-route bus services with flexible-route services and determined thresholds between them based mainly on demand density. These thresholds were then used in Chang and Schonfeld (1991b) to optimize systems that integrated these two service types. Parajuli and Wirasinghe (1991) proposed a two-stage approach for selecting transit modes by considering subjective factors and other cost parameters. Several more recent studies (Casello et al. 2014, Sivakumaran et al. 2014) also analyzed the selection of suitable transit modes, for given demand characteristics and financial concerns. Usually, a single transit mode was selected in those studies without considering the feeder-trunk structure.

Jara-Díaz and Gschwender (2003), Jara-Díaz et al. (2012) showed that a trunk-feeder service performs better than a single line when demands are spatially diversified. Chien and Schonfeld (1998) optimized such a rail-bus system and identified the benefit of schedule coordination at the transfer station. Other studies by Li et al. (2009) and Sivakumaran et al. (2014) also optimized such a service type from different perspectives. However, those 
methods were essentially static and incapable of revealing how the system service should be optimized in response to demand changes over an extended horizon. Cheng and Schonfeld (2015) presented a multi-period optimization model for the development of a rail transit line; nonetheless, the intermodal transfer was not considered. Problems such as how public transit modes should evolve with demand dynamics are still not fully solved.

The objective of this study is to find how the type of public transit services should evolve with demand over a planning horizon, while considering economies of scale in infrastructure construction and future cost discounting. The remainder of the paper is structured as follows: Section 2 presents the literature review on the selection of public transit modes and optimizations of the feeder-trunk service. A series of static models are formulated in Section 3 and a dynamic model is formulated in Section 4. A heuristic based on the tradeoffs between the initial and recurring cost is also proposed. Model applications are presented in Section 5, followed by main findings in Section 6.

\section{Literature Review}

\subsection{Selection of pure transit modes}

Several forms of public transport, also called public transit modes or technologies in the literature, are available, e.g., heavy rail, light rail and bus rapid transit. These differ in several ways, such as the capital cost required, operating cost, vehicle capacity, travel speed, and minimum headway. A systematic study by Tirachini et al. (2010) compares these three forms of public transit in terms of operator and users costs. Tirachini et al. (2010) suggest that in most cases a standard bus service outperforms a rail mode in terms of cost efficiency based on data from Australian cities. A few more studies are available on the selection of the optimal transit mode, i.e., identifying under what conditions a transit mode is preferred to other alternatives. Casello et al. (2014) present a method for selecting between the bus rapid transit and light rail transit through computing the life-cycle costs for candidate systems. In a case study conducted for Waterloo, Canada, they find there are no significant cost differences between two modes (bus rapid and light rail) and small changes in assumed inputs can reverse the preference. Sivakumaran et al. (2014) study the selection of transit mode for the trunk-line by considering the influence of its access mode. They find that the advantage of capital-intensive rail systems is underestimated if the transit is assumed to be accessed only by 
walking, as in most other studies, rather than by using other high-speed modes, e.g., bus rapid transit. Stutsman (2002) presents a case study of Los Angeles, CA, by comparing the bus rapid transit and the light rail in several aspects, namely, cost-effectiveness, environmental impact and community response.

Li et al. (2012) optimize a range of decision variables, such as the number of stations, headway and fare for a rail transit line in an urban corridor. The population density in the planning area is identified as the major factor in designing the rail transit line. The implications of the rail line length are also analyzed: a longer rail line provides greater coverage, while also incurring higher costs. Chen et al. (2015) adopt the modeling framework in Li et al. (2012) and study the transit mode selection instead. The critical population density is identified for selection of transit modes. Li et al. (2015) approach a similar problem as in Chen et al. (2015) in different ways. The uncertainty in population is explicitly considered by characterizing the population growth with a geometric Brownian motion. Considering the effect of transit mode choice on households' residential choice and housing market, they analytically derive the trigger population for implementing a transit mode. It should be noted that all these studies (Li et al. 2012, Chen et al. 2015, and Li et al. 2015) consider a single transit mode without examining the modal transfer. Chang and Schonfeld (1991a) analytically derive the threshold determining whether conventional or subscription bus feeder service is preferable for a region. That analysis is extended in Kim and Schonfeld (2013, 2014 and 2015) to determine which of those service types is preferable for various zones within a region and periods within a day, and to allocate buses accordingly.

\subsection{Optimization of the feeder-trunk transit service}

A single line (i.e. a pure transit mode) might be inefficient for serving spatially diversified demands and a trunk-feeder structure could be preferable to a single line (Jara-Díaz and Gschwender 2003). The relative advantages of corridors (with transfers) versus direct services (no transfers needed) are analyzed by Jara-Díaz and Gschwender (2003). Additional route structures, i.e., exclusive lines and shared lines, are compared to the single line and lines with transfers in a later study by Jara-Díaz et al. (2012). They identify under what conditions the feeder-trunk service dominate by assuming stylized passenger flows. However, the notion of schedule coordination is not mentioned in Jara-Díaz et al. (2012).

Chien and Schonfeld (1998) present one of the earliest systematical analyses of the rail-bus system. Decisions for the feeder service and trunk line, which include the rail line length, 
station spacing and headways, are jointly optimized. The benefit of schedule coordination is explicitly explored by comparing the coordinated and uncoordinated operations.

Such a trunk-feeder transit service is also optimized in more recent studies by Li et al. (2009), and Sivakumaran et al. (2014). Li et al. (2009) mainly examine the effect of market regime, i.e., monopoly (profit maximization), social optimum (social welfare maximization), and oligopoly, on the operation of a bus and rail transit system. Sivakumaran et al. (2014) show that the schedule coordination is Pareto-improving if frequencies of trunk and feeder services can be jointly optimized.

\subsection{Evolution of transit modes}

In this study, a dynamic model accounts for changes over time, i.e., time-dependent characteristics, while a static model considers only a steady state, i.e., time-invariant characteristics. Therefore, a static model is incapable of analyzing the evolution of modes. Chen et al. (2015) advance existing studies by identifying the timing of introducing the new transit mode in a simplified manner: the transit mode is introduced when the exponentially growing population density reaches the predefined threshold. Essentially, the modeling approach is still static. Li et al. (2015) adopt a real option approach to the timing problem by assuming the urban population follows a geometric Brownian motion. While significant contributions are made in these studies, no studies are available for optimizing the evolution of transit modes, e.g., the change of rail line length, over time. In a dynamic model, several practical considerations, such as economies of scale in construction and future cost discounting, should be included.

A multi-period model for extending the rail transit line is presented by Cheng and Schonfeld (2015). The implementation of a rail transit line is usually subdivided into several phases due to budget limits or insufficient demand. An optimization method is presented to subdivide a predesigned rail transit line into sections for phased development. However, only a single transit mode is considered in Cheng and Schonfeld (2015). In such a case, the rail line should be expanded as long as sufficient funding becomes available and demand warrants are met, because an expanded rail line represents a lower total system cost. As shown later, such a conclusion does not hold in a trunk-feeder system. There exists a minimum economic length of the rail line, meaning that any length below the threshold is suboptimal and should be avoided. 


\subsection{Summary}

We find the following based on the above literature review:

- Quite a few comparative analyses of various transit modes are available in the literature. With such methods, the optimal transit mode can be selected given demand characteristics, financial situations and other factors.

- It has been recognized that the line structure depends on the demand pattern. In case of spatially diversified demands, the trunk-feeder service is preferred to the single line (a pure technology). Several researchers have developed static models for optimizing the service parameters of the trunk-feeder transit service.

There are no dynamic models for analyzing the transition from a single line to a trunk-feeder structure over an extended horizon. Those static models which are used to identify the threshold to introduce the new mode are useful; however, they cannot solve the following problems effectively.

(1) What is the optimal rail line length when the bus only service is replaced by the rail-bus service?

(2) How does the optimal rail line length change when significant economies of scale and future cost discounting in the rail infrastructure construction are considered?

(3) How does the transit mode, which can be characterized by the rail line length as explained later, evolve with demand dynamics?

\section{Static Models}

\subsection{Assumptions}

The following simplifying assumptions are made:

(1) Originating travel demands are exogenously given and distributed along the commuter corridor stretching from the central business district (CBD) to the city boundary.

(2) All attractions are assumed to be located at the city center, implying that the demand pattern is many-to-one in this paper. This sacrifice of geographic detail helps us focus here on the relations among relevant factors. Such an assumption is relaxed in 
Appendix 1 by converting the origin-destination demand into the inflow, outflow and through flow for each location along a corridor.

(3) Demand is fixed in this study. Appendix 2 shows how this assumption can be relaxed to explicitly consider demand elasticity.

(4) The demand growth is assumed to be deterministic. When such an assumption does not hold, we can consider multiple future scenarios, as in Sun and Schonfeld (2015a), which complicate the model.

(5) Passengers located in regions inaccessible to rail must access rail stations by bus.

\subsection{User cost function}

The travel demand density $q(x)$ at location $x$ from the CBD is given by a linear function as follows:

$$
q(x)=\theta_{1}-\theta_{2} x, \quad \forall x \in(0, B]
$$

where $\theta_{1}$ and $\theta_{2}$ are known parameters. Particularly, when $\theta_{2}=0$ the demand distribution is uniform along the corridor. In Fig. 2, $L$ represents the length of the rail transit line, which is positive, and $B$ is the length of the corridor.

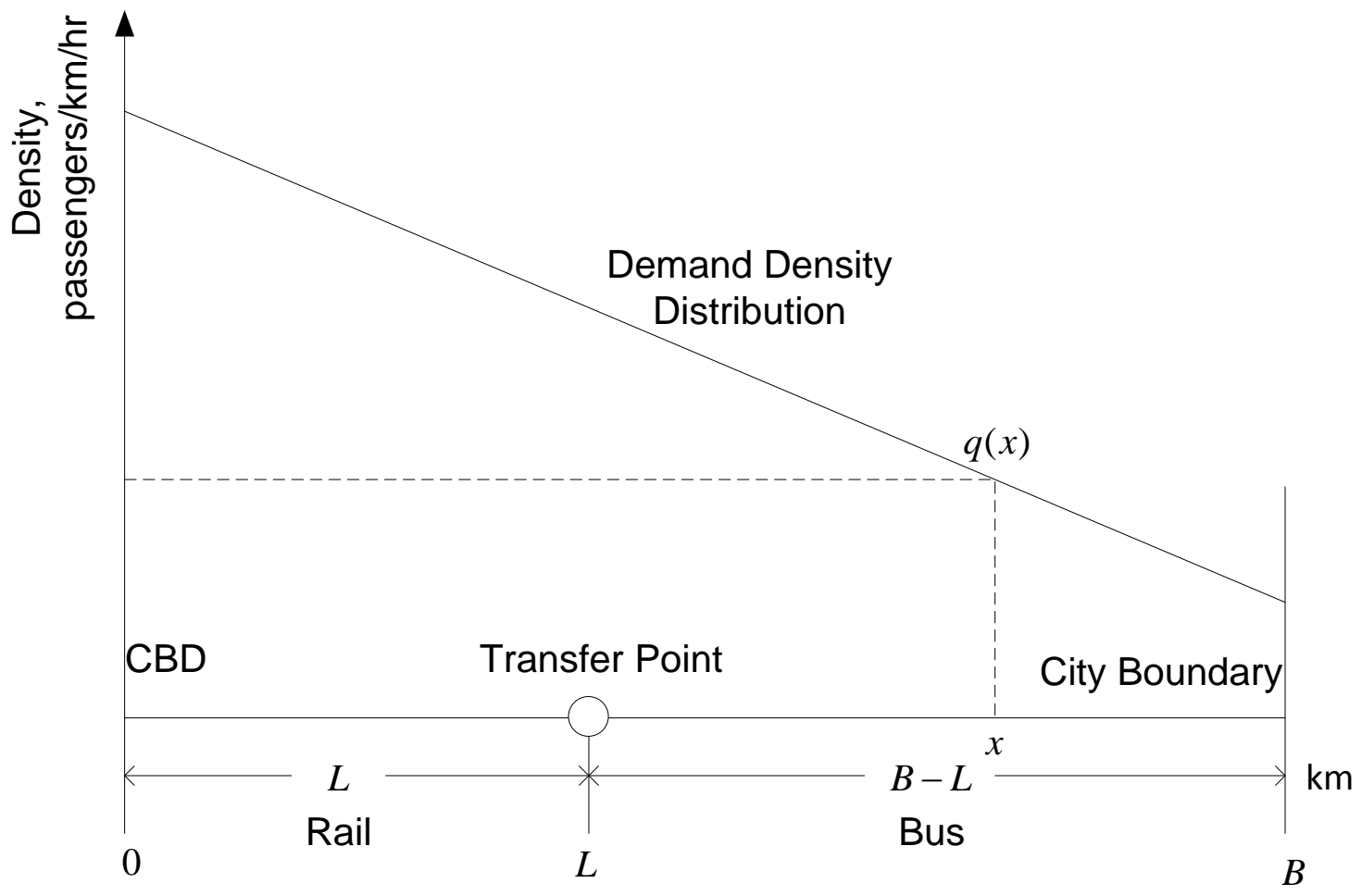

Fig. 2. Demand density distribution along the corridor 
$U(x)$ is the travel cost from location $x$ to the CBD (one way only) per person, which mainly depends on the distance traveled and service frequencies.

$$
U(x)=\left\{\begin{array}{l}
\tau \frac{x}{V_{1}}+\frac{e_{w}}{2} h_{1}+f_{1}, 0<x \leq \mathrm{L} \\
\tau\left(\frac{x-L}{V_{2}}+\frac{L}{V_{1}}\right)+\frac{e_{w}}{2} h_{1}+\frac{e_{w}}{2} h_{2}+f_{2}, L<x \leq B
\end{array}\right.
$$

where $\tau$ is the value of in-vehicle time, $e_{w}$ is the value of waiting time, $V_{1}$ is the average travel speed of trains, $V_{2}$ is the average travel speed of buses. $h_{1}$ and $h_{2}$ are headways of trains and buses. $f_{1}$ and $f_{2}$ are fixed costs for non-transferring passengers, i.e., taking trains only, and transferring passengers, respectively.

Generally, the average waiting time is half of the headway when passengers arrive at transit stops as a Poisson process, vehicle capacities are sufficient to not leave behind any passengers (Sun and Schonfeld 2015b), and vehicle headways are uniform and relatively short. In other cases, the average waiting time could be different. For example, when the headways are longer, e.g., more than 10-12 min, passengers do not arrive randomly, but consult pre-set schedules or real-time information before their trips. If vehicle arrivals are random, the waiting time also depends on the variance of headways, as in Osuna and Newell (1972). The total user cost can be calculated as follows:

$$
C_{u}=\int_{0}^{B} U(x) q(x) d x
$$

\section{Supplier Cost}

The operator cost is the hourly cost per vehicle (bus or train) multiplied by fleet size.

$$
C_{p}=\frac{2 b_{1}}{V_{1} h_{1}} L+\frac{2 b_{2}}{V_{2} h_{2}}(B-L)
$$

where $b_{1}$ and $b_{2}$ are the hourly costs per train and per bus, respectively.

$$
\begin{aligned}
& b_{1}=\alpha_{1}+\beta_{1} k_{1}, \\
& b_{2}=\alpha_{2}+\beta_{2} k_{2},
\end{aligned}
$$

where $\alpha_{1}$ and $\alpha_{2}$ are the fixed hourly costs for rail and bus, $\beta_{1}$ and $\beta_{2}$ are the marginal hourly costs per train-seat and bus-seat. $k_{1}$ and $k_{2}$ are capacities of trains and buses, i.e., numbers of seats. 
Four total cost minimization models are presented in the next for optimizing the uncoordinated rail and bus operations, rail operations only, bus operations only and coordinated operations. Since all those models are intended for operations optimization, the total cost obtained from such models is also referred to as "recurring cost", in contrast with "initial cost" for infrastructure construction. Note that here recurring cost includes the users' cost in addition to the operators' cost.

\subsection{Model 1 - Uncoordinated rail and bus operations}

In the first model, decisions are optimized independently for the rail and bus segments. The service type is feeder-trunk with only one feeder route beyond the end of the rail line.

The objective is to minimize the sum of operators' cost and users' cost, subject to the bus and train capacity constraints.

$$
\begin{aligned}
\min _{\left\{h_{1}, h_{2}, k_{1}, k_{2}>0\right\}} C_{T} & =\int_{0}^{L}\left(\tau \frac{x}{V_{1}}+\frac{e_{w}}{2} h_{1}+f_{1}\right) q(x) d x+\int_{L}^{B}\left(\tau\left(\frac{x-L}{V_{2}}+\frac{L}{V_{1}}\right)+\frac{e_{w}}{2} h_{1}+\frac{e_{w}}{2} h_{2}+f_{2}\right) q(x) d x \\
& +\frac{2\left(\alpha_{1}+\beta_{1} k_{1}\right)}{V_{1} h_{1}} L+\frac{2\left(\alpha_{2}+\beta_{2} k_{2}\right)}{V_{2} h_{2}}(B-L)
\end{aligned}
$$

subject to

$$
\begin{aligned}
& h_{1} \int_{0}^{B}\left(\theta_{1}-\theta_{2} x\right) d x \leq k_{1} \eta_{1}, \\
& h_{2} \int_{L}^{B}\left(\theta_{1}-\theta_{2} x\right) d x \leq k_{2} \eta_{2},
\end{aligned}
$$

where $\eta_{1}, \eta_{2}$ are load factors for rail and bus.

By noting that the operator cost $C_{p}$ is an increasing function in $k_{1}$ and $k_{2}$, we conclude that there is no need to provide excess capacity, i.e., Eqs (8) and (9) are binding. If the congestion effect is considered, e.g., the comfort level decreases fast if the ratio of demand to capacity grows, such binding constraints do not hold.

Letting $Q_{1}=\int_{0}^{B}\left(\theta_{1}-\theta_{2} x\right) d x$ and $Q_{2}=\int_{L}^{B}\left(\theta_{1}-\theta_{2} x\right) d x$, we can insert $k_{1}=h_{1} Q_{1} / \eta_{1}$ and $k_{2}=h_{2} Q_{2} / \eta_{2}$ into the objective (7). The total cost becomes a function of headways only. We set the first-order partial derivatives of the objective with regard to $h_{1}$ and $h_{2}$ to be zero.

$$
\frac{\partial C_{T}}{\partial h_{1}}=\frac{e_{w}}{2} \int_{0}^{L}\left(\theta_{1}-\theta_{2} x\right) d x+\frac{e_{w}}{2} \int_{L}^{B}\left(\theta_{1}-\theta_{2} x\right) d x-\frac{2 L \alpha_{1}}{V_{1} h_{1}^{2}}=0
$$




$$
\frac{\partial C_{T}}{\partial h_{2}}=0+\frac{e_{w}}{2} \int_{L}^{B}\left(\theta_{1}-\theta_{2} x\right) d x-\frac{2(B-L) \alpha_{2}}{V_{2} h_{2}^{2}}=0,
$$

Due to the nonnegativity of headways, we obtain:

$$
\begin{aligned}
& h_{1}^{*}=2 \sqrt{\frac{L \alpha_{1}}{V_{1} e_{w} Q_{1}}}, \\
& h_{2}^{*}=2 \sqrt{\frac{(B-L) \alpha_{2}}{V_{2} e_{w} Q_{2}}},
\end{aligned}
$$

Due to binding capacity constraints, we further have:

$$
\begin{aligned}
& k_{1}^{*}=\frac{2}{\eta_{1}} \sqrt{\frac{L \alpha_{1} Q_{1}}{V_{1} e_{w}}}, \\
& k_{2}^{*}=\frac{2}{\eta_{2}} \sqrt{\frac{(B-L) \alpha_{2} Q_{2}}{V_{2} e_{w}}},
\end{aligned}
$$

Since the closed form solutions of headways and vehicle capacities are available, they can be inserted into the objective (7), which becomes a function of only one decision variable $L$, which is the length of the rail line:

$$
\begin{aligned}
\min _{\{0<L<B\}} & \int_{0}^{L}\left(\tau \frac{x}{V_{1}}+\sqrt{\frac{e_{w} L \alpha_{1}}{V_{1} Q_{1}}}+f_{1}\right) q(x) d x+ \\
& \int_{L}^{B}\left(\tau\left(\frac{x-L}{V_{2}}+\frac{L}{V_{1}}\right)+\sqrt{\frac{e_{w} L \alpha_{1}}{V_{1} Q_{1}}}+\sqrt{\frac{e_{w}(B-L) \alpha_{2}}{V_{2} Q_{2}}}+f_{2}\right) q(x) d x+ \\
& \sqrt{\frac{e_{w} L \alpha_{1} Q_{1}}{V_{1}}}+\sqrt{\frac{e_{w}(B-L) \alpha_{2} Q_{2}}{V_{2}}}+\frac{\beta_{1} Q_{1}}{\eta_{1}} \sqrt{\frac{e_{w} L Q_{1}}{V_{1} \alpha_{1}}}+\frac{\beta_{2} Q_{2}}{\eta_{2}} \sqrt{\frac{e_{w}(B-L) Q_{2}}{V_{2} \alpha_{2}}}
\end{aligned}
$$

The new objective only has one decision variable; however, the functional form is rather complex. Finding a closed form solution of the optimal length $L^{*}$ by setting the first-order derivative to be zero seems difficult. Therefore, one can adopt a numerical evaluation method over the whole region $(0, B)$ to find the optimized rail length when train operation and bus operation are uncoordinated.

\subsection{Model 2 - Rail only}

Since in Model 2 the rail transit line covers the entirety of the corridor, the rail length is $B$. The optimization model is:

$$
\min _{\left\{h_{1}, k_{1}>0\right\}} C_{T}=\int_{0}^{B}\left(\tau \frac{x}{V_{1}}+\frac{e_{w}}{2} h_{1}+f_{1}\right) q(x) d x+\frac{2\left(\alpha_{1}+\beta_{1} k_{1}\right)}{V_{1} h_{1}} B
$$


subject to

$$
h_{1} \int_{0}^{B}\left(\theta_{1}-\theta_{2} x\right) d x \leq k_{1} \eta_{1}
$$

Following the same method used to solve Model 1, we obtain:

$$
\begin{aligned}
& h_{1}^{*}=2 \sqrt{\frac{B \alpha_{1}}{V_{1} e_{w} Q_{1}}}, \\
& k_{1}^{*}=\frac{2}{\eta_{1}} \sqrt{\frac{B \alpha_{1} Q_{1}}{V_{1} e_{w}}},
\end{aligned}
$$

\subsection{Model 3 - Bus only}

Replacing the subscript " 1 " with " 2 ", one can obtain the optimization model and results for bus only operations. The rail line length is zero.

\subsection{Model 4 - Coordinated rail and bus operations}

In Model 1 we assume that bus and train operations are uncoordinated. At the transfer point, when the bus's arrival is independent from the train's departure, all transferring passengers face additional waiting for the train. When the bus schedule and train schedule are coordinated, passengers can seamlessly transfer between buses and trains, thus greatly reducing the additional waiting time. One simple way to achieve the schedule coordination or timed transfer is to use a common headway or integer-ratio headways (Ting and Schonfeld 2005). In this study, we further assume that when $h_{1}: h_{2}=1: 1$, i.e., the common headway $h_{0}$ is used, the transfer waiting time is negligible

The user cost function is thus modified into:

$$
U^{\prime}(x)=\left\{\begin{array}{l}
\tau \frac{x}{V_{1}}+\frac{e_{w}}{2} h_{0}+f_{1}, 0<x \leq \mathrm{L} \\
\tau\left(\frac{x-L}{V_{2}}+\frac{L}{V_{1}}\right)+\frac{e_{w}}{2} h_{0}+f_{2}, L<x \leq B
\end{array}\right.
$$

The total cost minimization model for coordinated operations is:

$$
\begin{aligned}
\min _{\left\{h_{0}, k_{1}, k_{2}>0\right\}} C_{T} & =\int_{0}^{L}\left(\tau \frac{x}{V_{1}}+\frac{e_{w}}{2} h_{0}+f_{1}\right) q(x) d x+\int_{L}^{B}\left(\tau\left(\frac{x-L}{V_{2}}+\frac{L}{V_{1}}\right)+\frac{e_{w}}{2} h_{0}+f_{2}\right) q(x) d x \\
& +\frac{2\left(\alpha_{1}+\beta_{1} k_{1}\right)}{V_{1} h_{0}} L+\frac{2\left(\alpha_{2}+\beta_{2} k_{2}\right)}{V_{2} h_{0}}(B-L)
\end{aligned}
$$


subject to

$$
\begin{aligned}
& h_{0} \int_{0}^{B}\left(\theta_{1}-\theta_{2} x\right) d x \leq k_{1} \eta_{1}, \\
& h_{0} \int_{L}^{B}\left(\theta_{1}-\theta_{2} x\right) d x \leq k_{2} \eta_{2},
\end{aligned}
$$

Similarly, we obtain the following relations analytically:

$$
\begin{aligned}
& h_{0}^{*}=2 \sqrt{\frac{L \alpha_{1}}{V_{1} e_{w} Q_{1}}+\frac{(B-L) \alpha_{2}}{V_{2} e_{w} Q_{1}},} \\
& k_{1}^{*}=\frac{2}{\eta_{1}} \sqrt{\frac{L \alpha_{1} Q_{1}}{V_{1} e_{w}}+\frac{(B-L) \alpha_{2} Q_{1}}{V_{2} e_{w}}}, \\
& k_{2}^{*}=\frac{2}{\eta_{2}} Q_{2} \sqrt{\frac{L \alpha_{1}}{V_{1} e_{w} Q_{1}}+\frac{(B-L) \alpha_{2}}{V_{2} e_{w} Q_{1}}},
\end{aligned}
$$

\section{Dynamic Model}

With the static models, one can decide what combination of transit modes, i.e., rail plus bus (uncoordinated operations), rail only and bus only, is the most cost effective from the perspective of operations, for a given demand input. Since each mode can be characterized by the unique variable, namely the length of the rail segment, by noting that in Model 1, $0<L<B$; in Model 2, $L=B$; in Model 3, $L=0$, one can find the optimized rail length, which minimizes the total cost, for each given demand distribution. If the demand growth curve over a planning horizon is assumed to known, a dynamic problem can be formulated.

\subsection{Assumptions}

The following assumptions are made to facilitate the formulation.

(1) Time is treated as being continuous.

(2) The future demand growth is deterministic.

(3) There are no backlogs in demands.

While the first two assumptions are self-explanatory, more discussion is warranted for Assumption (3). In the capacity expansion literature, there are at least two approaches: (a) capacity leads demand and (b) capacity lags demand. In (a), capacity is added before the demand warrants, i.e., no backlogs in demand are allowed. As illustrated in Fig. 3, this expansion strategy results into excess capacity, which indicates a higher cost (Sun and 
Schonfeld 2015a). However, excess capacity can help operators make more flexible decisions and it can absorb disruptions. Therefore, it is commonly used in practice. Intuitively, if approach (b) is adopted instead, the capacity curve lies below the demand forecast.

This line extension problem is analogous to the general capacity expansion problem. For any given demand input, the optimal transit mode or a combination of modes can be found from the operations perspective. The optimal mode choice, which is characterized simply by the length of the rail segment, is thus the optimal "capacity" warranted by the given demand. Analogously, rail expansion leads the demand requirement in this study.

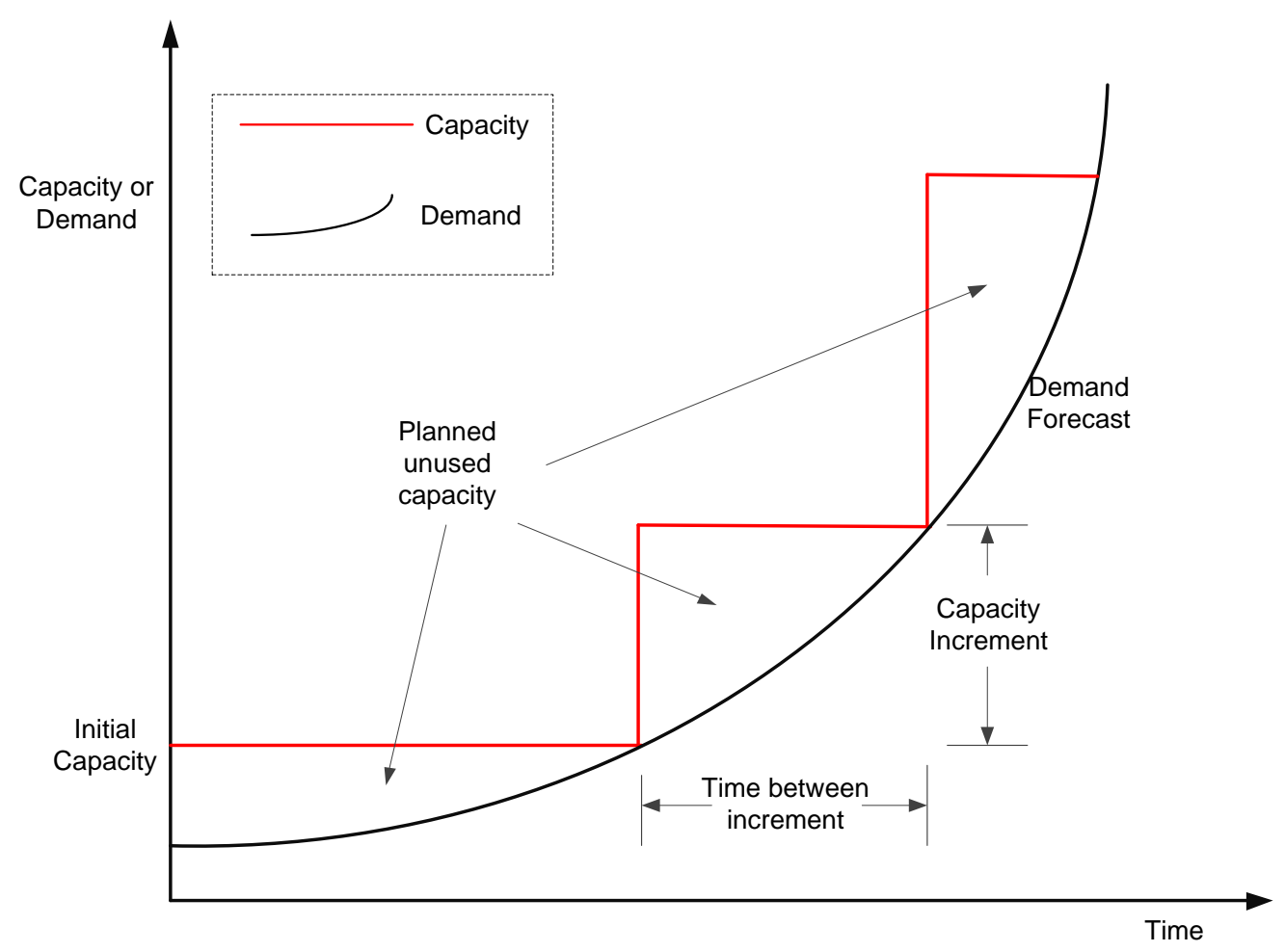

Fig. 3 Capacity expansion strategy - capacity leads demand

\subsection{Formulation}

Fig. 4 illustrates some notations. The time horizon is denoted as $T$. At time $t \in T$, the demand distribution is $d_{t}=\left(\theta_{1}^{t}, \theta_{2}^{t}\right)$. A binary variable $z_{t}$ is used to indicate whether the rail line is extended at $t \in T$ and a nonnegative variable $x_{t}$ represents the extended length. If $z_{t}=0$, the rail line is not extended, i.e., $x_{t}=0$; if $z_{t}=1$, the extended length $x_{t}$ could be 
positive, incurring a fixed cost of $K_{0}$. At $t$, the capital cost of extending the rail by the length of $x_{t}$ is defined as:

$$
I_{t}=K_{0} z_{t}+K_{1} x_{t}, t \in T
$$

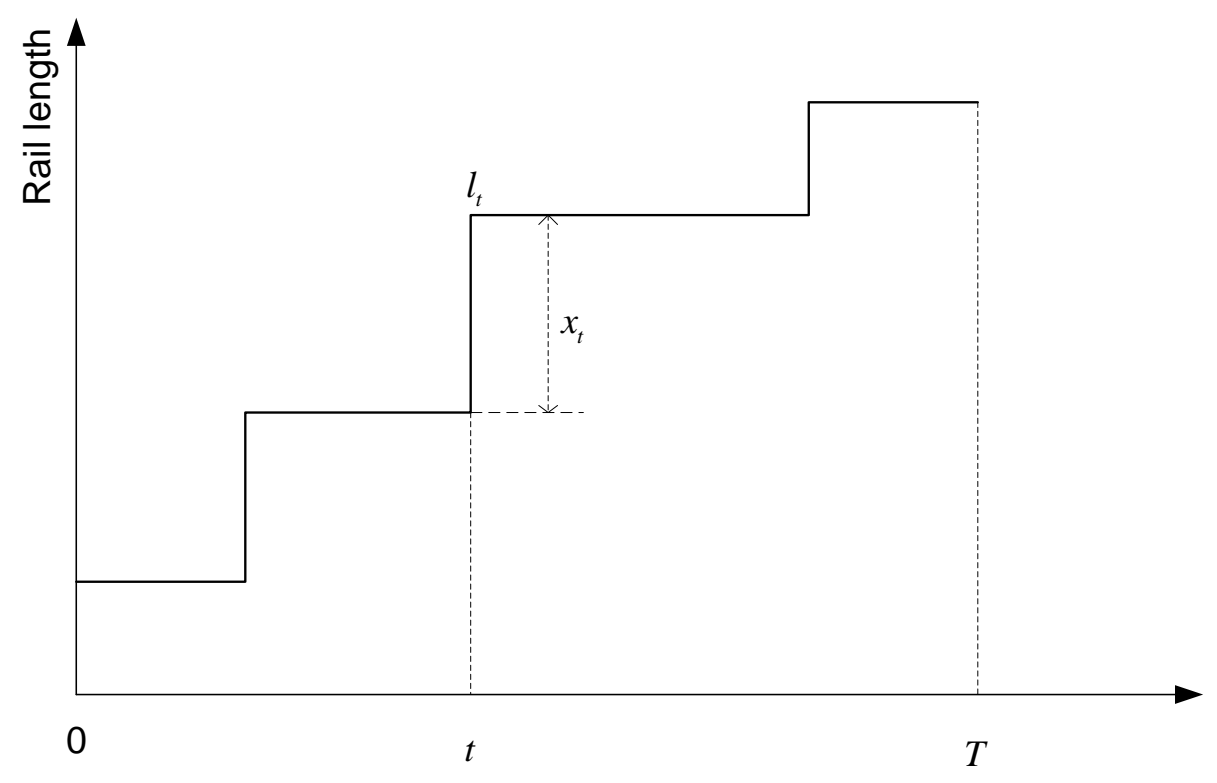

Fig. 4 Definitions of symbols

The operations cost obtained from the static model, e.g., Model 1 , is $C_{T}\left(l_{t}, d_{t}\right)$. It depends on the length of the rail segment and the demand distribution. Note that $C_{T}(\cdot)$ is evaluated on an hourly basis and $N_{p}$ is used to convert it to the units used in defining the planning horizon. If the horizon length is measured in years, $N_{p}$ is the number of equivalent operating hours in one year.

By considering the fixed cost of extending the rail, economies-of-scale have been included in the model. Another essential factor in long-term planning problems is future cost discounting. We adopt the expression $e^{-\gamma t}$ as the conversion coefficient, where $\gamma$ is a discount rate, e.g., 0.03 or 0.05 . When $\gamma=0.03$ and $t=20, e^{-\gamma t}=0.55$, meaning that the present value of capital cost could be reduced by almost a half if the same extension is postponed from $t=0$ to $t=20$.

The objective of the dynamic model is to minimize the discounted cost of the capital cost associated with the extension $I_{t}\left(x_{t}\right)$ and the operational cost $C_{T}\left(l_{t}, d_{t}\right)$ over the horizon. The dynamic model is formulated as: 


$$
\min _{\left\{x_{t} \geq 0, z_{t} \in\{0,1\}\right\}} \int_{0}^{T} e^{-\gamma t}\left(N_{p} * C_{T}\left(l_{t}, d_{t}\right)+I_{t}\right) d t,
$$

subject to

$$
\begin{aligned}
& \int_{0}^{y} x_{t} d t=l_{y}, y \in T \\
& x_{t} \leq M z_{t}, t \in T
\end{aligned}
$$

Constraint (30) defines how the length of the rail segment is accumulated. Constraint (31) restricts that $x_{t}$ can be positive only when $z_{t}=1$. The symbol $y$ also represents a time in the planning horizon, which is used interchangeably with $t$. The expression of $C_{T}\left(l_{t}, d_{t}\right)$ is in objective (16) and Equation (28) defines $I_{t}$.

\subsection{Solution Algorithm}

The proposed dynamic model contains both discrete and continuous decision variables. Although the capital cost is linear in $x_{t}, C_{T}\left(l_{t}, d_{t}\right)$ is a nonlinear function of $l_{t}$, which is a linear combination of $x_{t}$. The mathematical expression of the objective is quite complex, and its derivative seems unlikely to be derivable analytically. Therefore, the model combines the difficulties of solving a mixed integer program, a nonlinear program, and a derivate-free optimization problem. Nonetheless, by exploring the structure of the model, we find the solution is in fact a staircase function defined over the horizon. The number of steps and the step size determine the tradeoffs between the initial and recurring costs.

From the perspective of operations, the rail line should be extended to the exact length warranted by the demand input at a given time. Such a solution, which is shown as a curve in Fig 5(a), represents the minimum length requirement. Such a continuous extension plan is only possible when the fixed cost of an extension is extremely small, i.e., almost zero. It is also referred to as "frictionless investment" in the capacity expansion literature (Van Mieghem 2003). Conversely, when the fixed cost is extremely large, according to Assumption (3), the rail line should be extended to its maximal length, as defined by the highest possible demand in one step. The reason is that the fixed cost is too large and extensions in multiple steps are not economically justified. Such a solution represents the one-time extension. 

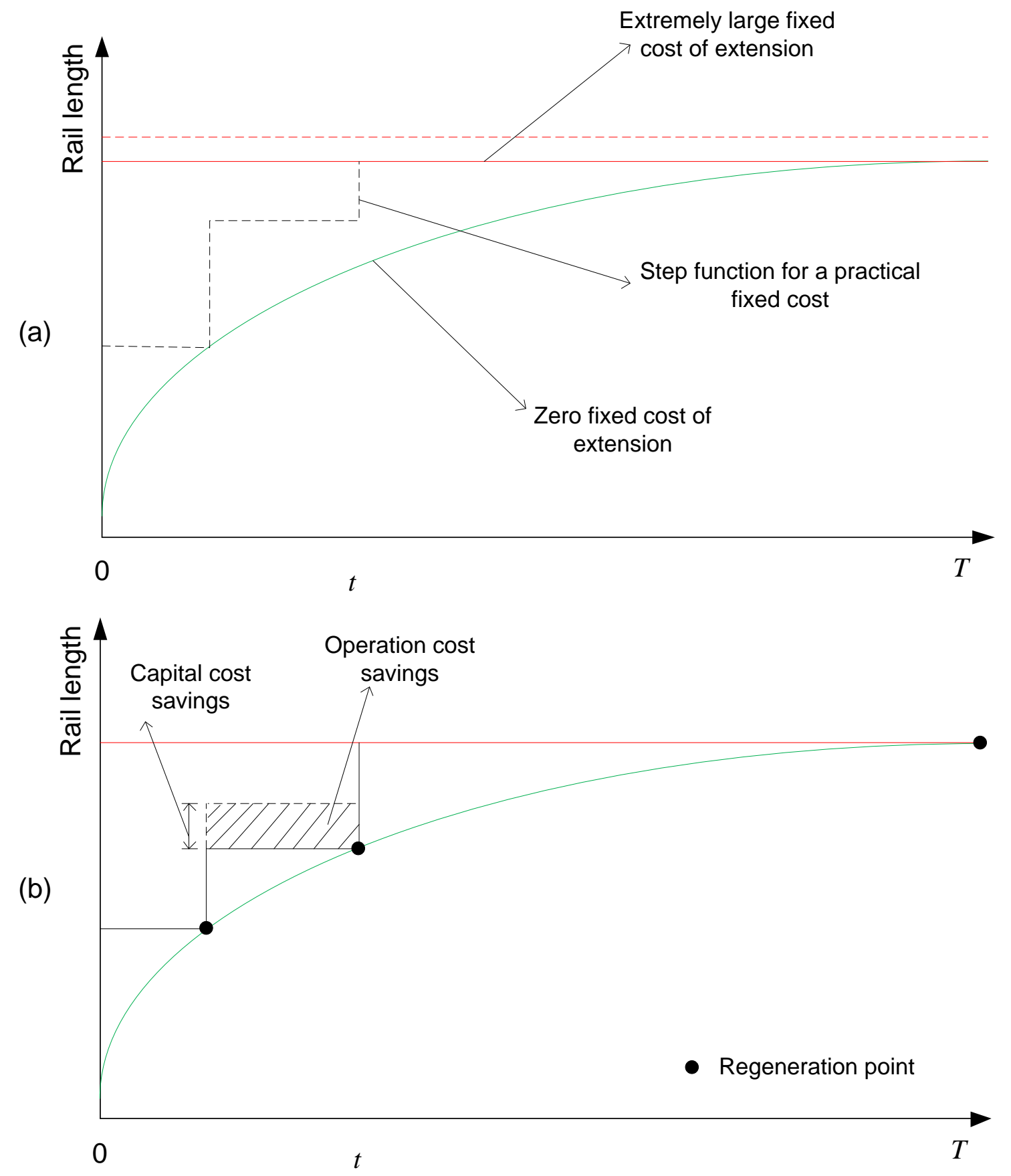

Fig. 5 Illustration of solution dominance

We first show that the one-time extension and the continuous extension have one point of intersection. As in Fig. 5(a), if the dashed flat line is considered as a candidate optimal solution, we find by moving the line downwards until it touches the continuous extension curve, the objective is improving because (1) the capital cost is lowered and (2) the excess rail line is shorter, which indicates operational savings. Therefore, there is no need to extend the line beyond the limit required by the highest demand. Such an intersection is called a "regeneration point", which means the excess rail line length becomes zero. 
For a practical value of the fixed $\operatorname{cost} K_{0}$, the optimal solution is bounded by the one-time extension and continuous extension. We further show that at the time of extension, the excess length is zero, implying a regeneration point exists. In Fig 5 (a), the rail length at time $t$ is excessive. If this extension plan (dashed step function in Fig. 5 (a)) is modified into the solid step function in Fig. 5 (b), there are cost savings in two aspects. Partial length is added in later periods, meaning a lower present value of the capital cost. In the period starting from the prior extension to $t$, the excess length is reduced, representing additional savings from operations. Therefore, the dashed step function is dominated by the solid one. Then we conclude that there is a regeneration point at each extension. Time 0 is an exception because that is the initial construction, not an extension. Based on the above discussions, we can find the step function is uniquely determined by a set of regeneration points.

A heuristic is proposed for finding the optimized solution, as shown in Fig. 6.

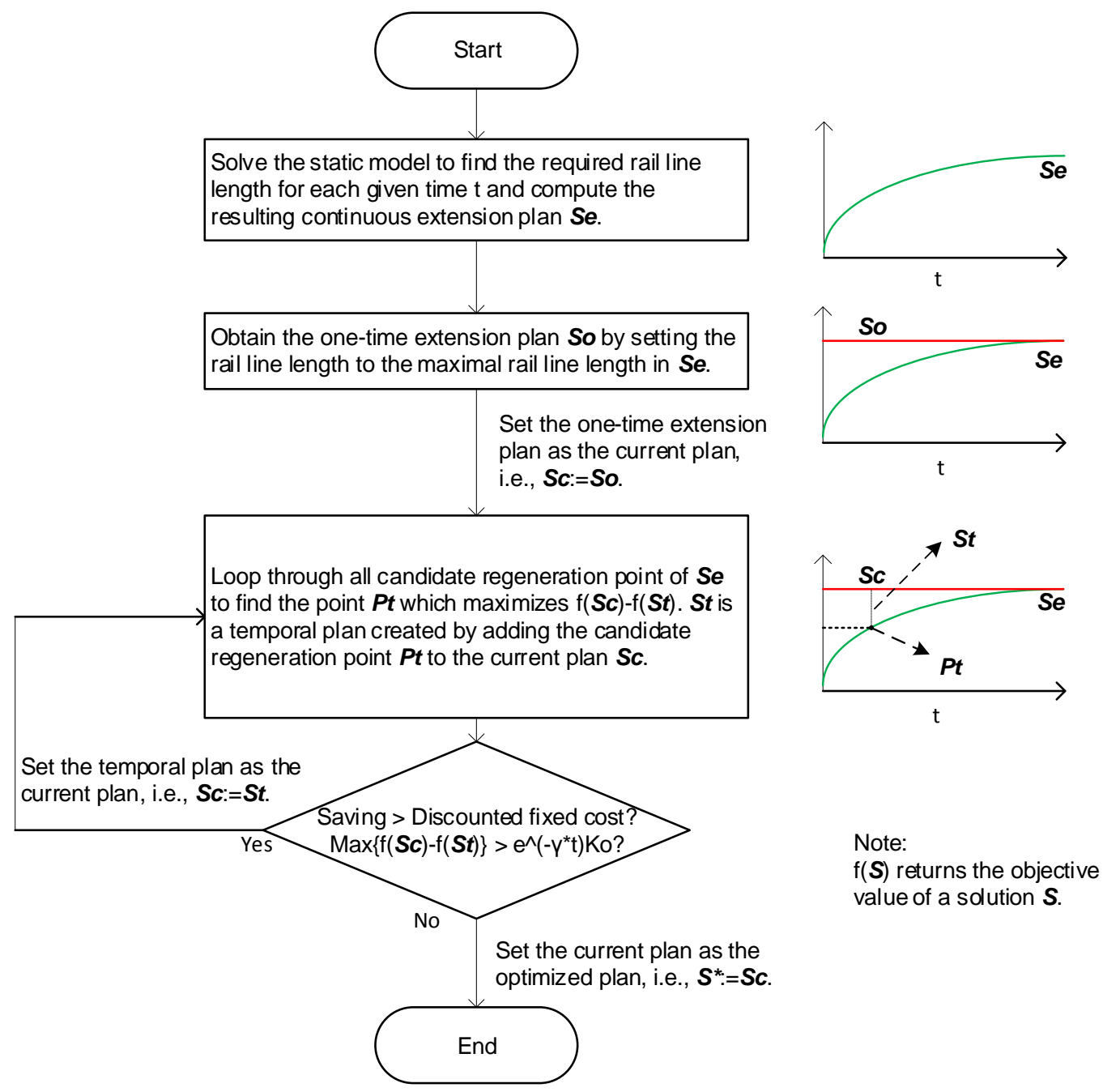

Fig. 6 Flowchart of the Proposed Graphical Solution Method 


\section{Model Applications}

The data in Table 1 are used in the following numerical analyses to demonstrate the model.

TABLE 1 Baseline values

\begin{tabular}{|c|c|c|c|}
\hline Notations & Definitions & Baseline Values & Unit \\
\hline$B$ & Corridor length & 50.0 & mi \\
\hline$\tau$ & Value of in-vehicle travel time & 10 & $\$ / \mathrm{hr}$ \\
\hline$e_{w}$ & Value of waiting time & 40 & $\$ / \mathrm{hr}$ \\
\hline$V_{1}$ & Average travel speed - Rail & 40 & $\mathrm{mi} / \mathrm{hr}$ \\
\hline$V_{2}$ & Average travel speed - Bus & 30 & $\mathrm{mi} / \mathrm{hr}$ \\
\hline$\alpha_{1}$ & Fixed hourly cost - Rail & 1500 & $\$ / \mathrm{hr}$ \\
\hline$\alpha_{2}$ & Fixed hourly cost - Bus & 120 & $\$ / \mathrm{hr}$ \\
\hline$\beta_{1}$ & Variable hourly cost - Rail & 3.0 & $\$ / \mathrm{hr} /$ seat \\
\hline$\beta_{2}$ & Variable hourly cost - Bus & 6.0 & $\$ / \mathrm{hr} / \mathrm{seat}$ \\
\hline$\eta_{1}$ & Load factor - Rail & 1.2 & -- \\
\hline$\eta_{2}$ & Load factor - Bus & 1.1 & -- \\
\hline$f_{1}$ & Fixed component of use costs - rail & 1.0 & $\$$ \\
\hline$f_{2}$ & Fixed component of use costs - bus & 1.0 & $\$$ \\
\hline$N_{p}$ & Operation hours per year & 3600 & -- \\
\hline$T$ & Length of the planning horizon & 20.0 & years \\
\hline$K_{0}$ & Fixed capital cost of rail extension & $0.1-3.0$ & Million \$ \\
\hline$K_{1}$ & Marginal capital cost per mile & 2.0 & Million \$ \\
\hline$r$ & Yearly discounting rate & 0.03 & -- \\
\hline
\end{tabular}

\subsection{Interior solution of the optimal rail length}

It has been shown that operation parameters, i.e., headways and vehicle capacities for bus and rail, are determined by the rail length $L$ when the demand distribution $q(x)$ is given. In Model 1, objective (16) is a function of the only decision variable $L$ and a numerical search 
over the range $(0, B)$ can be used to find the optimized $L^{*}$.

Fig. 7 shows the optimization results for each demand distribution, which is characterized by the density at $\operatorname{CBD} \theta_{1}$ and the ratio of the density at the end of the corridor and the density at CBD, i.e., $\left(\theta_{1}-B \theta_{2}\right) / \theta_{1}$, which is specified in the legend. Particularly, when the ratio is 1.0 , the demand density is uniform along the corridor.

From Fig. 7, the rail length should increase when the ratio increases for a given $\theta_{1}$ because a larger ratio means a larger passenger volume, as shown in Fig. 8. The optimal rail increases as $\theta_{1}$ increases for a given ratio; however, the curve becomes quite flat with respect to $\theta_{1}$, which means the rail line should not be extended to the city limit for such a transportation corridor. In Fig. 8, the circle on each curve represents the corresponding location of the transfer point.

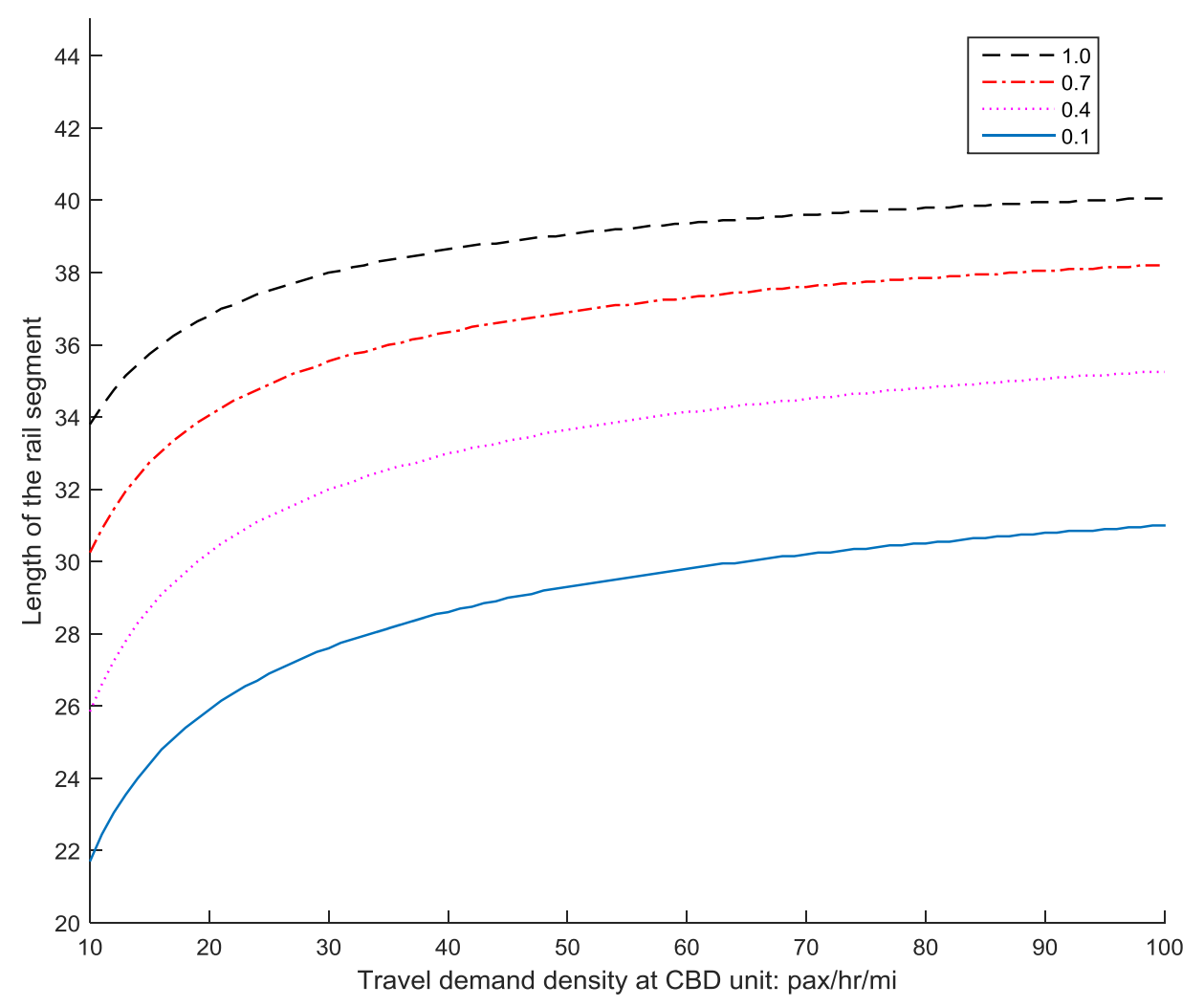

Fig. 7. Optimized rail length vs given demand 


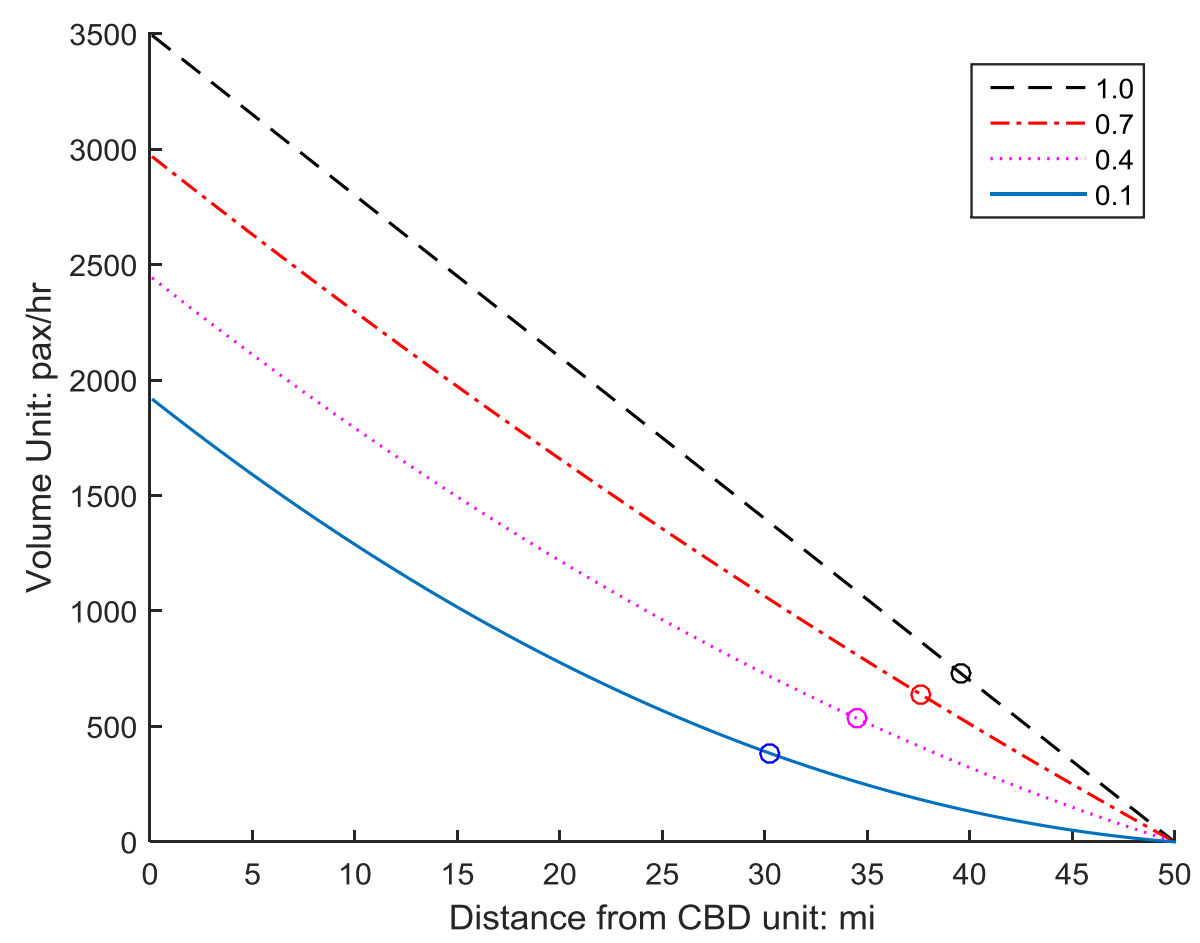

Fig. 8. Aggregate passenger volume and locations of the transfer point

The method only searches over the open interval $(0, B)$ and does not check the endpoints, namely 0 and B. In fact, $L=0$ or $L=B$ are undefined in Model 1. The next subsection shows the potential pitfalls of using such an interior solution approach.

\subsection{Corner solution of the optimized rail length}

The interior solution approach is valid when the transit mode or the combination of modes is fixed. In the above analysis, only the service type of rail plus bus, i.e., Model 1, is studied and no alternatives, e.g., Model 2 and Model 3, are compared. A comparison in terms of the average trip cost reveals under what conditions which mode is the best choice, as in Fig. 9. The ratio $\left(\theta_{1}-B \theta_{2}\right) / \theta_{1}$ is 0.1 in Fig. 9. Overall, the average trip cost decreases as the demand density grows, indicating the economies of scale in transit operations. The average trip cost is the total system cost divided by the number of trips. The curve for Model 3 intersects the curve for Model 1. At the intersection point (called the "threshold" in Chang and Schonfeld (1991)) of the curve for Model 3 and the curve for Model 1, one is indifferent between bus operation only and the hybrid operations of rail and bus. In other words, the hybrid operations dominate the pure bus operation only beyond this intersection. Before this intersection, the optimal length should be zero, i.e., bus only, as indicated in Fig. 9. If only the interior solution method is used and corner solutions are unchecked, invalid optimization 
results (red segment in Fig. 10) can be obtained because in a certain range, other technologies than the one assumed might be preferable.

The pure bus operation can dominate the hybrid operation; it seems that the pure rail operation is always dominated by the hybrid one. In other words, even when the demand density is very high, a combined rail and bus alternative is more cost efficient than rail only. This is valid because even when the origin trip density is uniform, the resulting trip volume passing through a certain location is non-uniform, with peaks in the urban area and lows in the suburban area.

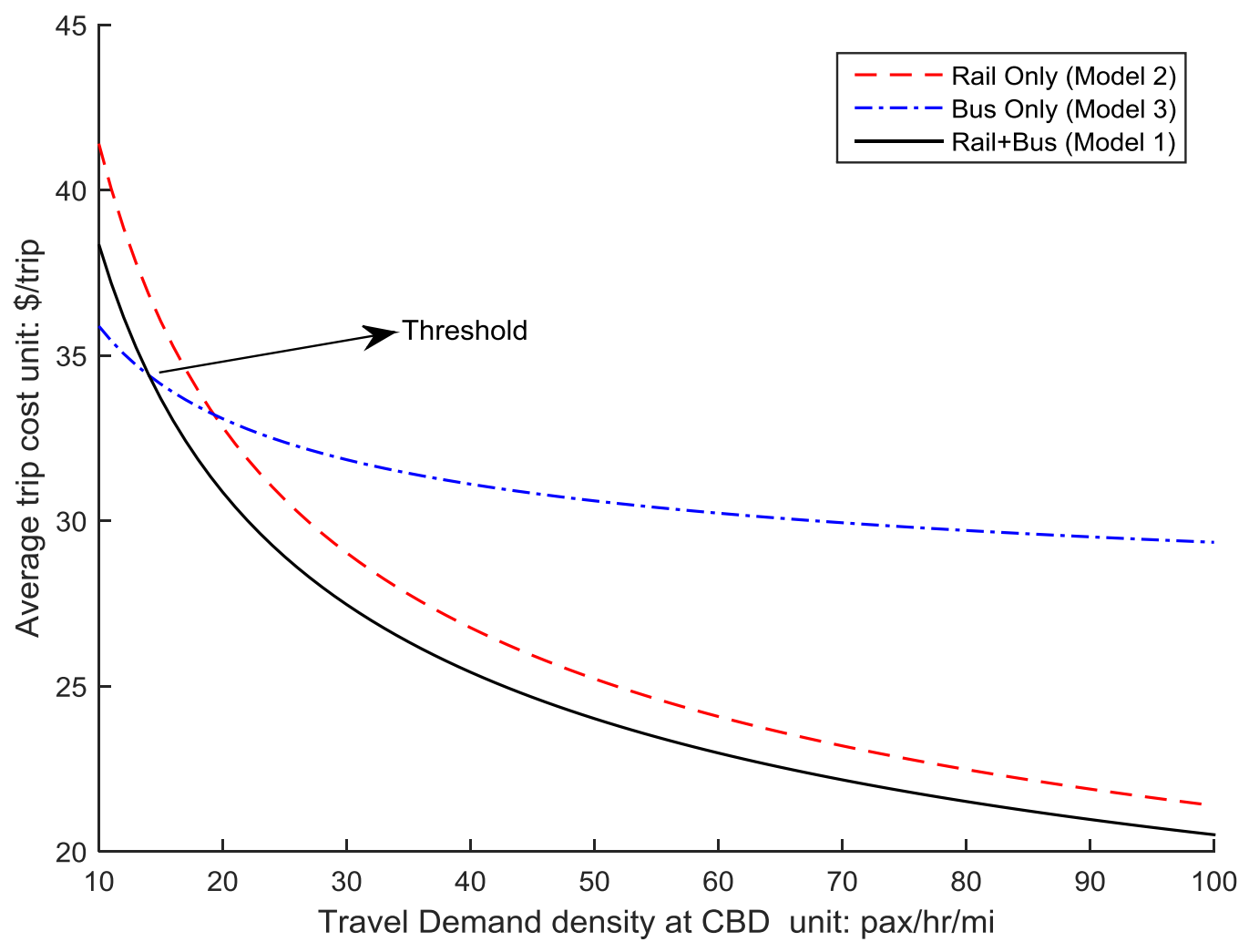

Fig. 9. Comparisons of three choices of transit technologies 


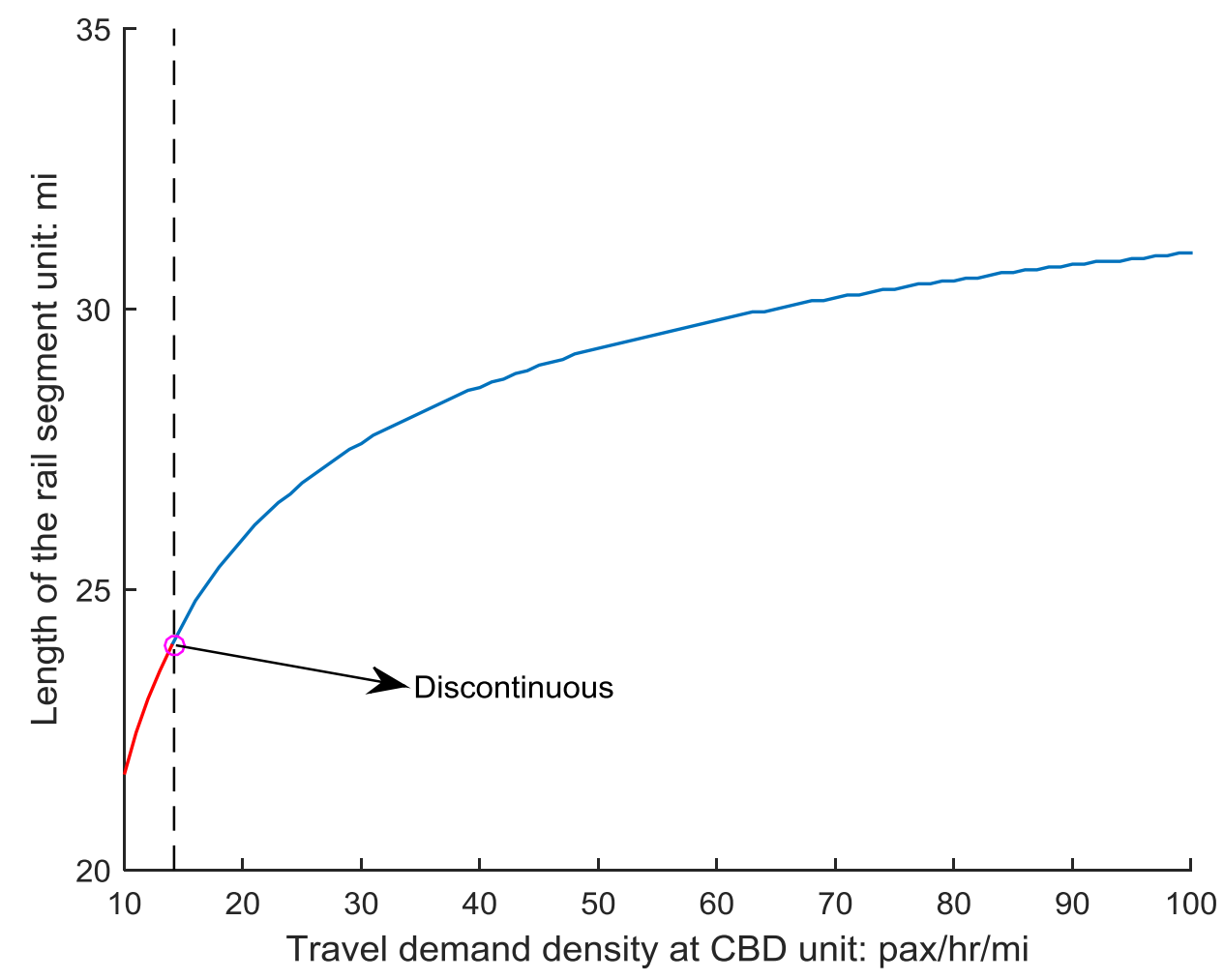

Fig. 10. Discontinuous rail length

\subsection{Effect of timed transfers}

Model 1 and Model 4 are compared by assuming the same rail length $L=30.2$ and demand distribution $\left(\theta_{1}=70, \theta_{2}=1.26\right)$. The comparison results are presented in Table 2 . The common headway is larger than either the bus headway or the train headway in the independent operations. We find the same result by comparing Eq. (25) with Eqs (12) and (13). Since the demand to be served is the same, the vehicle sizes required in the coordinated operation are significantly larger. Due to such a change, users' costs increase while operators' costs decrease. The net effect is that the total cost could be saved by $0.15 \mathrm{e} 4 \$ / \mathrm{hr}$ by adopting the common headway. Nonetheless, such a benefit is not guaranteed. If random factors drive the transit operations off the predetermined schedule, the coordinated transfer is disrupted and such a cost saving may be reduced or forfeited.

In fact, it is not necessary to use a common headway. An integer-ratio headway can also be considered, as shown in Ting and Schonfeld (2005). 
Table 2 Comparison of uncoordinated and coordinated operations

\begin{tabular}{|l|c|c|c|}
\hline Notations & Uncoordinated & Coordinated & Remark \\
\hline$h_{1}$ & 0.24 & 0.28 & Rail headway hr \\
\hline$h_{2}$ & 0.14 & 0.28 & Bus headway hr \\
\hline$k_{1}$ & 389 & 452 & Train capacity \\
\hline$k_{2}$ & 50 & 99 & Rus capacity \\
\hline$C_{u}^{1}$ & $1.41 \mathrm{e}+04$ & $1.53 \mathrm{e}+04$ & Bus riders' cost $\$ / \mathrm{hr}$ \\
\hline$C_{u}^{2}$ & $7.27 \mathrm{e}+03$ & $6.47 \mathrm{e}+03$ & Train operating cost $\$ / \mathrm{hr}$ \\
\hline$C_{p}^{1}$ & $1.66 \mathrm{e}+04$ & $1.53 \mathrm{e}+04$ & Bus operating cost $\$ / \mathrm{hr}$ \\
\hline$C_{p}^{2}$ & $3.88 \mathrm{e}+03$ & $3.34 \mathrm{e}+03$ & $C_{T}=C_{u}^{1}+C_{u}^{2}+C_{p}^{1}+C_{p}^{2}$ \\
\hline$C_{T}$ & $4.19 \mathrm{e}+04$ & $4.04 \mathrm{e}+04$ & \\
\hline
\end{tabular}

\subsection{Dynamic Extension}

As illustrated in Fig. 10, the economic length of the rail line is around 24 miles if inputs are specified as in subsection 5.2. To interpret the so-called economic length, any rail line shorter than the threshold is not cost-effective because such a feeder-trunk service with a short rail segment is dominated by the bus-only service. Therefore, the evolution of the rail line is examined only beyond the threshold. The starting point of evolution is Time 0 , when one is indifferent between the bus only service and the feeder-trunk structure. Before Time 0 , the rail length is 0 , which is not shown in the following analyses.

At Time $0, \theta_{1}=14.2$ passengers $/ \mathrm{hr} / \mathrm{mi}$ and the linear growth rate is 10 per year. For convenience, the ratio $\left(\theta_{1}-B \theta_{2}\right) / \theta_{1}$ is kept as 0.1 . It is worth noting that any other demand growth patterns can be used; these would affect the determination of the continuous extension plan.

First, a relatively small fixed cost $K_{0}=0.1 e 6$ is used. The following regeneration points on the continuous extension curve are added sequentially: [6.5, 2.5, 11.9, 1.1, 4.3, 15.5, 8.7]. The element in the vector represents the timing. Except for the regeneration point at the end of the continuous extension curve, the cost saving due to adding a regeneration point, i.e., one more 
extension, is shown in Fig. 11. Whenever a regeneration point is added, the fixed cost is incurred. If the cost saving (see Fig. 5) due to adding of the regeneration point exceeds the fixed cost, the addition is justified.

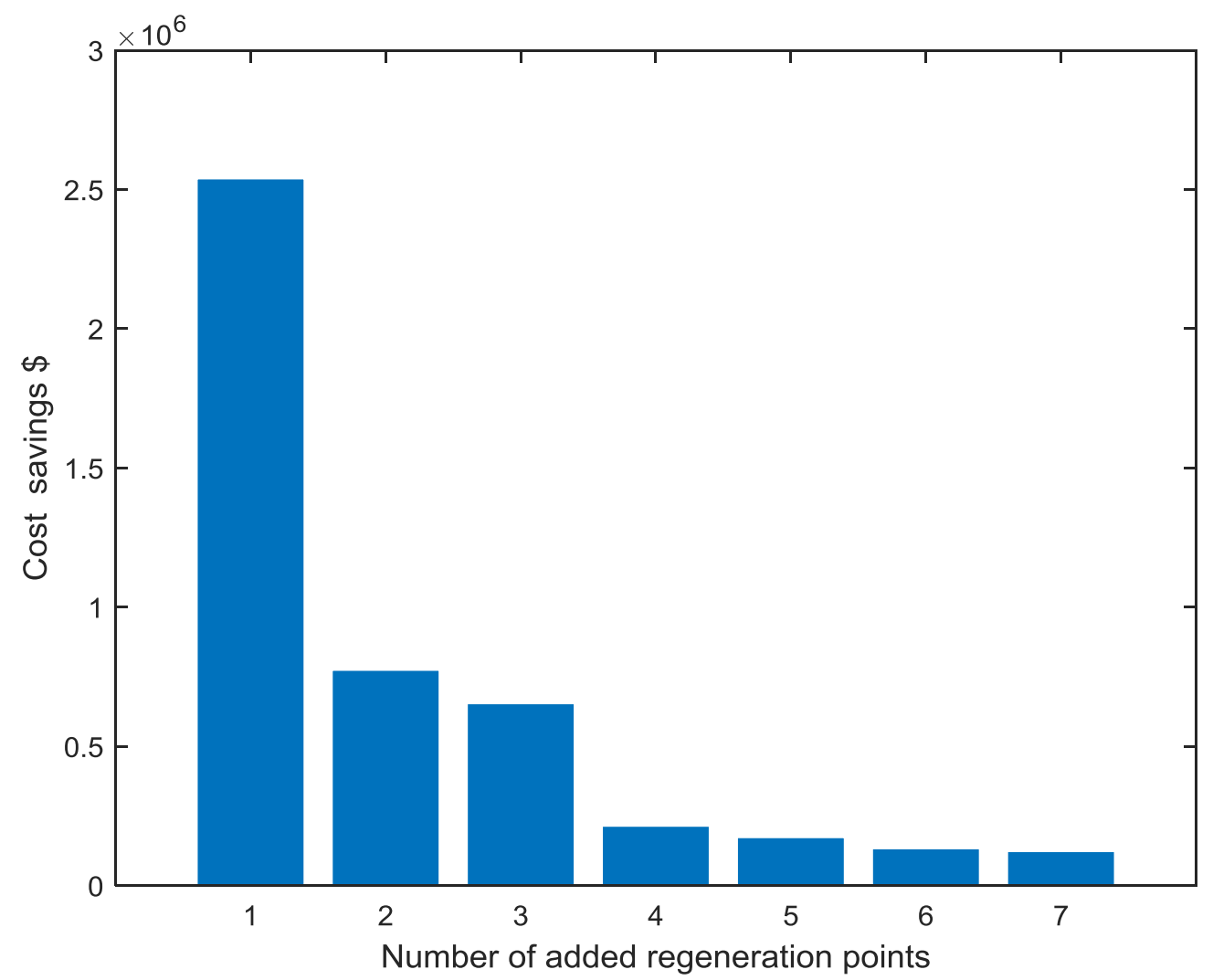

Fig. 11 Cost saving in each iteration as a regeneration point is added

Fig. 12 shows how a new solution is gradually generated by cutting off a rectangular region one at a time. The resulting extension plan is illustrated with the step function in Fig. 13. 


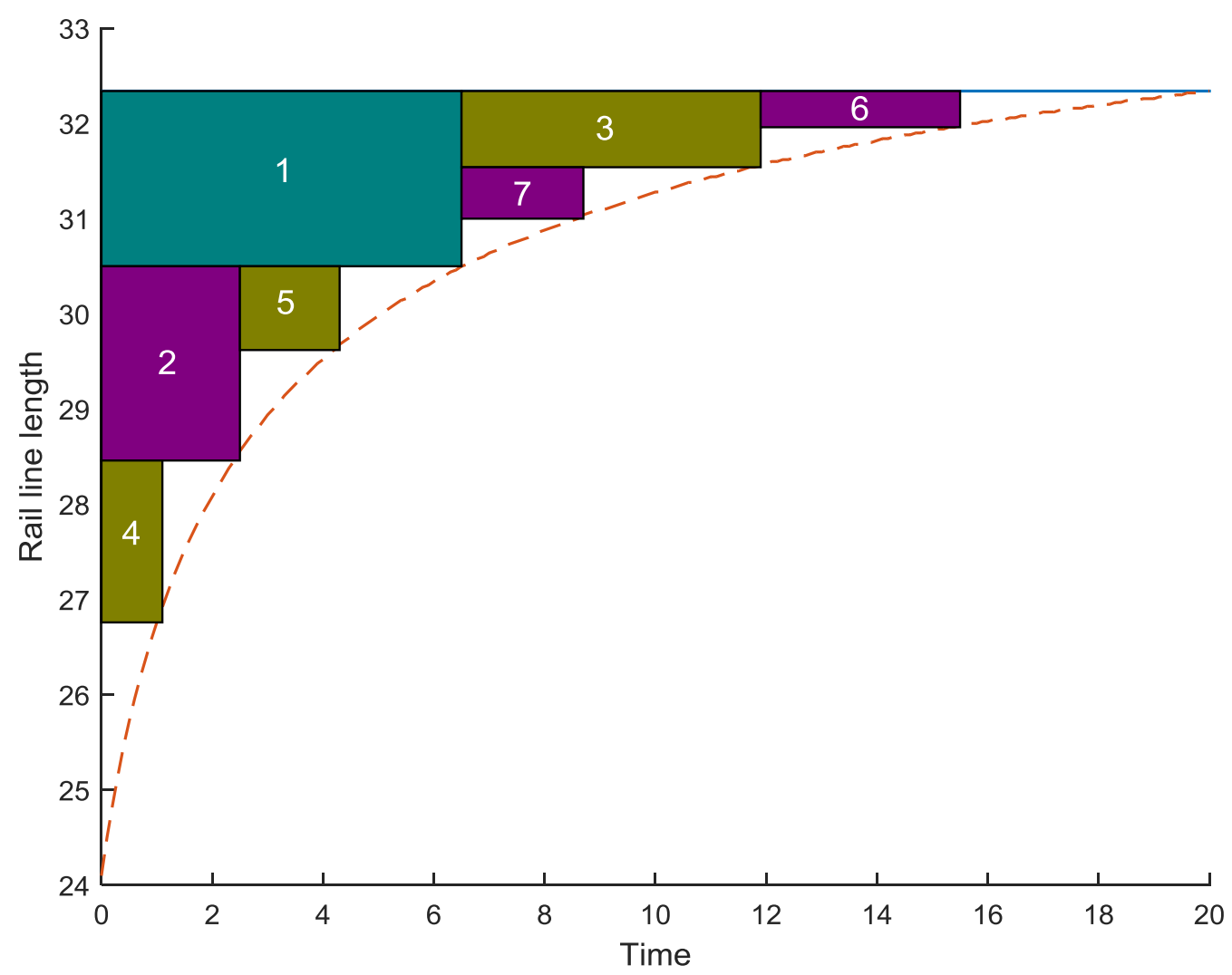

Fig. 12 Gradual reductions of excess supplied capacities

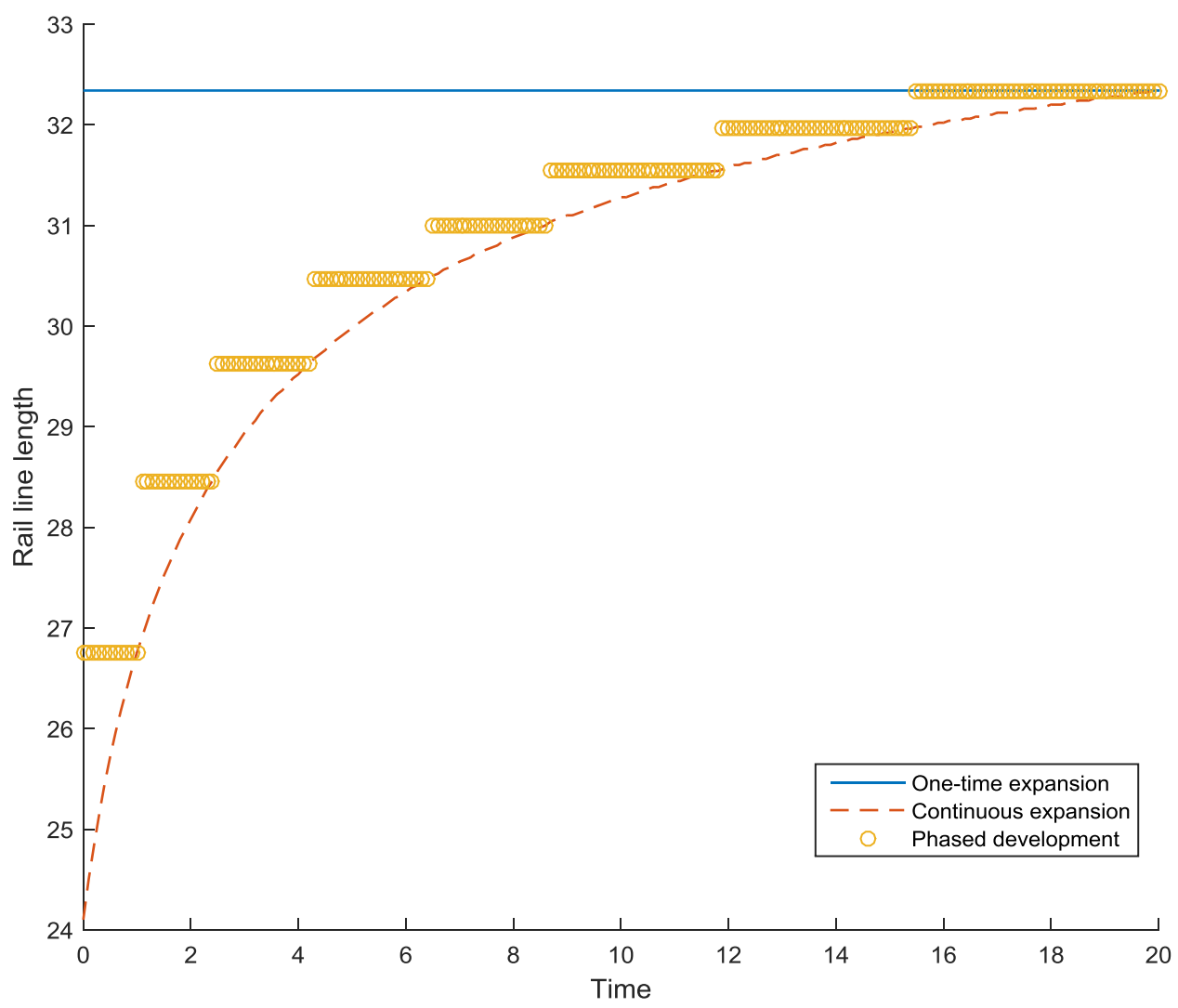

Fig. 13 Evolution of rail line length over time $\left(K_{0}=0.1 e 6\right)$ 
It is also found that the initial rail length (roughly $26.8 \mathrm{mi}$ in Fig. 13) is even longer than the economic length (around $24.0 \mathrm{mi}$ in Fig. 10) identified through static models. Such an additional length arises from the consideration of economies of scale.

When larger values of $K_{0}$ are used, the extension takes place in fewer steps in Fig. 14 and Fig. 15. The space between the step function and the continuous extension curve becomes larger, meaning the excess supplied capacity. Scale economies in the capital cost lead to the line extension dynamics involving rather large changes. When the fixed cost is even larger the maximal possible cost saving (around 2.51e6 according to Fig. 11) by adding a regeneration point, the extension occurs in one single step, as shown in Fig. 16. The effect of economies of scale on the rail line evolution is revealed by comparing Figs. 13, and 14-16.

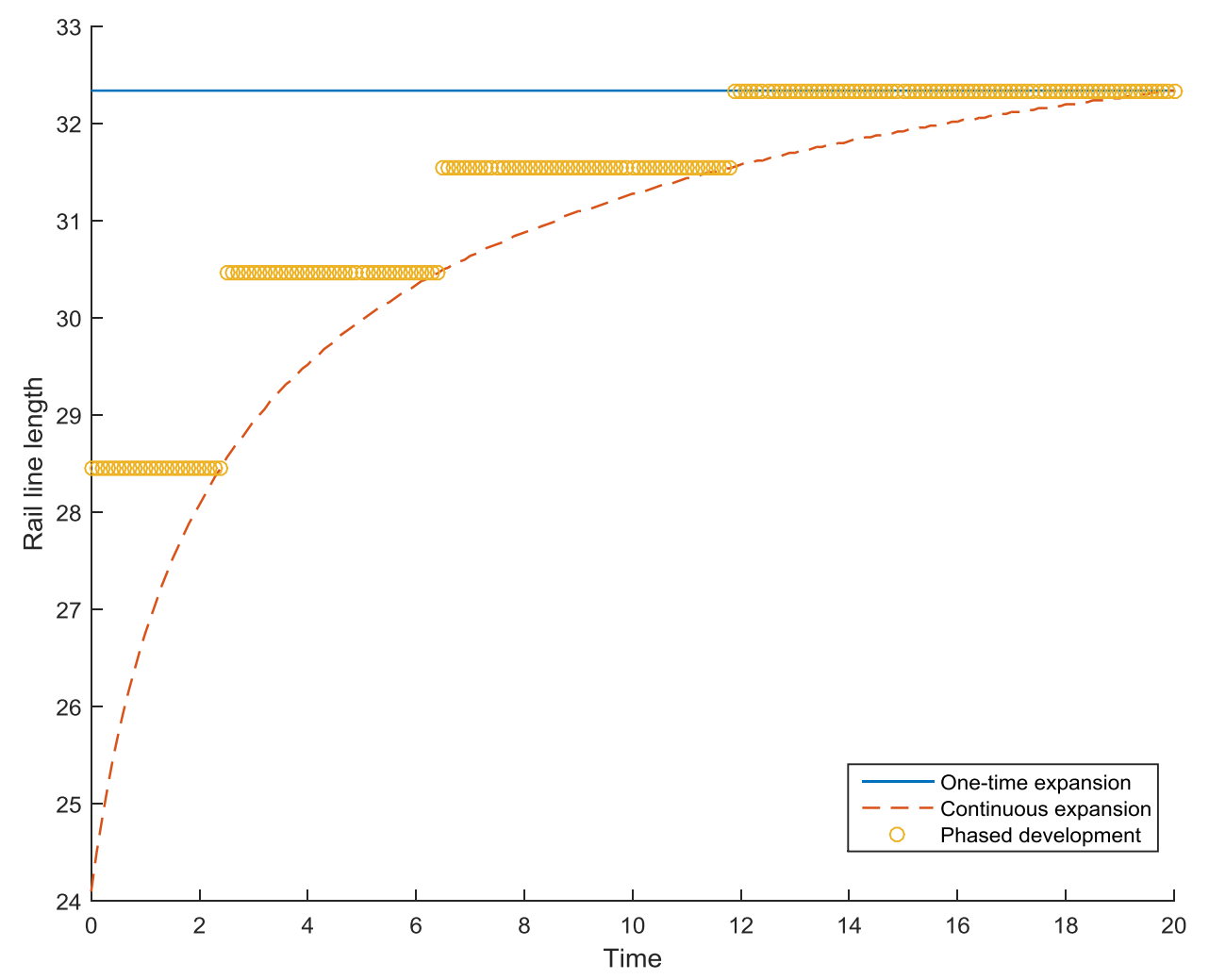

Fig. 14 Evolution of rail line length over time $\left(K_{0}=0.5 e 6\right)$ 


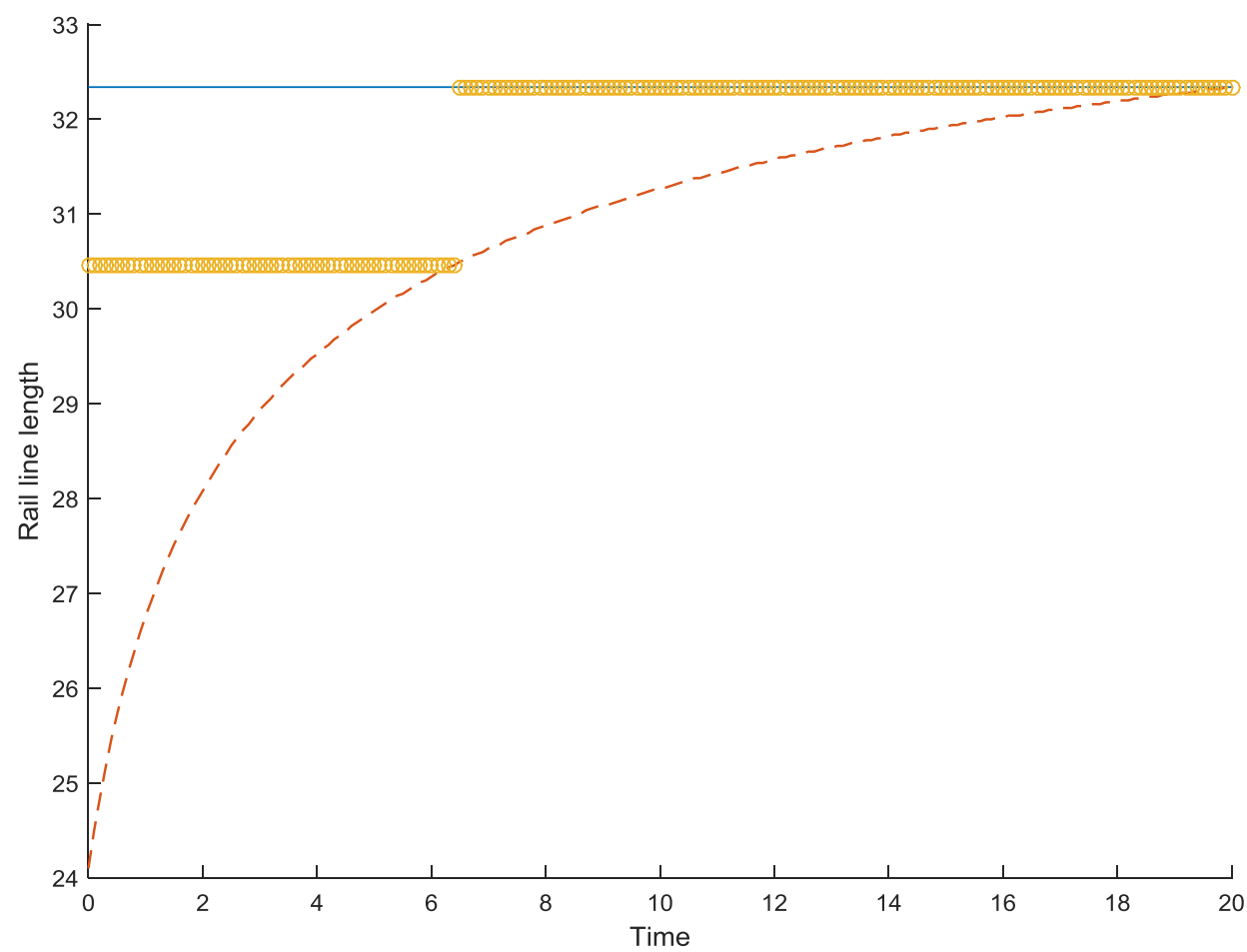

Fig. 15 Evolution of rail line length over time $\left(K_{0}=1.0 e 6\right)$

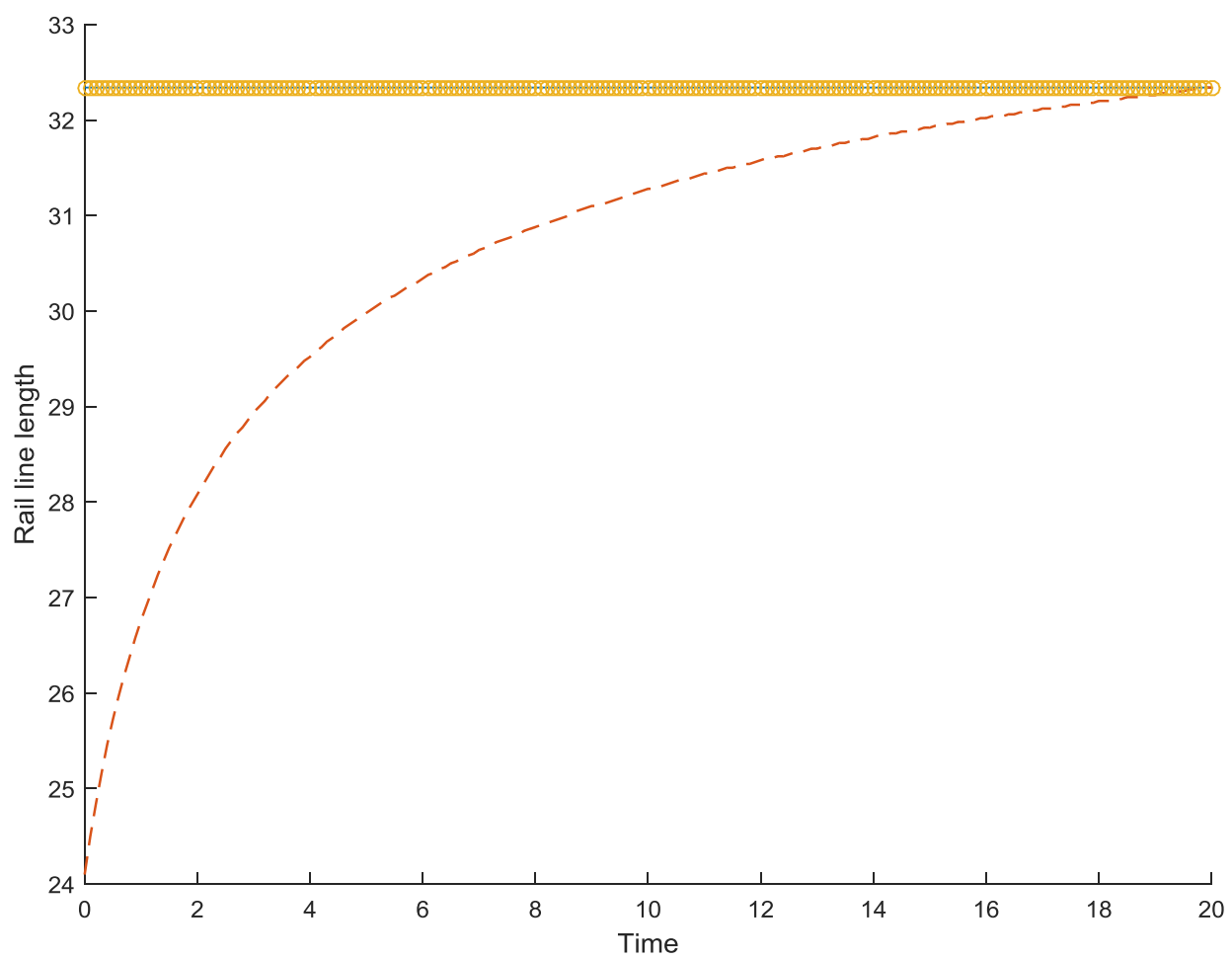

Fig. 16 Evolution of rail line length over time $\left(K_{0}=3.0 e 6\right)$ 


\section{Conclusions}

A series of static models, namely total cost minimization models for uncoordinated bus and rail operations, rail only, bus only, and coordinated bus and rail operations, are formulated and solved for a commuter corridor. The type of transit service is characterized by the length of the rail line. For a given demand input, the optimal length of the rail line is thus found. The pitfalls of not checking the boundary solutions are also illustrated. After noting there are very few dynamic models which are capable of examining the evolution of public transit services over an extended period, a dynamic model is formulated considering scale economies and future cost discounting. A graphical solution method based on the tradeoffs between the initial and reoccurring costs are used to find the optimized solution. The effect of the fixed cost parameter on the evolution of the rail transit line is revealed with case studies.

Major findings include the following:

(1) There exists a minimum economic length of the rail line in the feeder-trunk system, which can be determined numerically.

(2) Economies of scale lead to relatively large extensions and excess supplied capacity.

(3) The rail-only service is largely dominated by the feeder-trunk service even in the long run.

(4) The rail length may be erroneously determined if only interior solutions are searched.

One advantage of the continuum approximation approach is that analytically tractable solutions can be obtained, possibly in closed forms. Although simplifications are made in the derivation, these obtained analytical results can reveal important relations among various factors. For example, the conceptual analysis shows that there is an economic length of rail line, because a feeder-trunk service with a shorter rail segment than the threshold is dominated by the bus-only service.

However, this model has several limitations and may be improved in the following aspects.

(1) Geographic characteristics are simplified in this conceptual study to highlight the relations among important factors. This present work should be extended to consider more realistic real-world cases.

(2) The proposed heuristic is in fact a method of steepest descent, which usually leads to a local minimum. Other optimization methods may be considered, but it is unlikely that global optimality can be guaranteed in more complex and realistic case studies.

(3) Uncertainty in demand forecasts can be considered. 
(4) The access cost is neglected because the space is continuous. In practice, bus stops and train stations are located discretely. In such a case, the access cost should be part of the user cost.

(5) The present model is designed for a single corridor and can be extended to consider a multi-corridor or network problem.

(6) Other competing modes such as driving should be considered and a mode split model can be introduced.

\section{Acknowledgement}

The helpful comments from four anonymous reviewers and guest editors are gratefully acknowledged. 


\section{Appendix 1 - Analysis with Many-to-Many Demand Pattern}

The demand pattern assumed in the study is quite simple. More realistic demand characteristics can be considered. Passengers can travel to multiple destinations, not necessarily all located in the CBD, thus creating a many-to-many demand pattern. Fig. A-1 (a) illustrates such a pattern. Travelers are allowed to travel from any origin $x_{o}$ to destination $x_{d}$. The hourly volume of the Origin-Destination (OD) pair $\left(x_{o}, x_{d}\right)$ is denoted as $q\left(x_{o}, x_{d}\right)$. We require $0 \leq x_{d}<x_{o} \leq B$. For some OD pairs which satisfy $L<x_{o} \leq B$ and $0 \leq x_{d}<L$, passengers have to make a transfer at the location $L$. Such a trip can be broken into two legs as shown in Fig. A-1 (b). Then, the volume of such an OD pair is added two other OD pairs, namely $\left(x_{o}, L\right)$ and $\left(L, x_{d}\right)$, and the original OD pair is dropped out of the analysis.

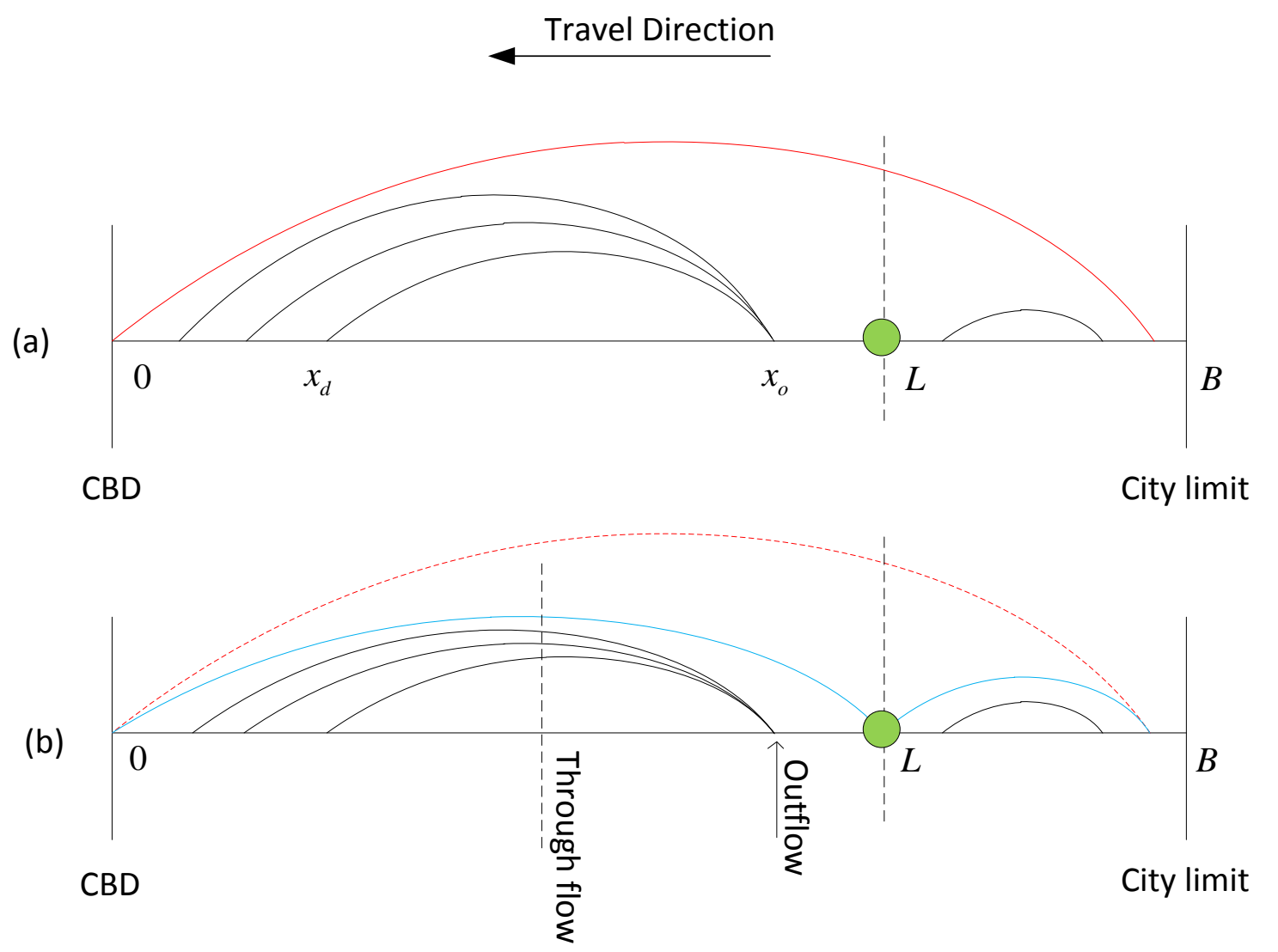

Fig. A-1. Demand density distribution along the corridor

The transfer point divides the corridor into two independent regions, namely rail line (superscript "1") and bus route (" 2 "). The passenger volume leaving from location $x \in(0, L]$ is:

$$
Q_{\text {out }}^{1}(x)=\int_{0}^{x} q\left(x, x_{d}\right) d x_{d}, \quad \forall x \in(0, L]
$$


The volume passing through location $x \in(0, L]$ is:

$$
Q_{T r u}^{1}(x)=\int_{0}^{x} \int_{x}^{L} q\left(x_{o}, x_{d}\right) d x_{o} d x_{d}, \quad \forall x \in(0, L]
$$

Similarly, the passenger volume leaving from location $x \in(L, B]$ is:

$$
Q_{\text {Out }}^{2}(x)=\int_{L}^{x} q\left(x, x_{d}\right) d x_{d}, \quad \forall x \in(L, B]
$$

The volume passing through location $x \in(L, B]$ is:

$$
Q_{T r u}^{2}(x)=\int_{L}^{x} \int_{x}^{B} q\left(x_{o}, x_{d}\right) d x_{o} d x_{d}, \quad \forall x \in(L, B]
$$

The optimization model for uncoordinated bus and rail operations is:

$$
\begin{aligned}
\min _{\left\{h_{1}, h_{2}, k_{1}, k_{2}>0\right\}} C_{T} & =\int_{0}^{L} \int_{0}^{x}\left(\tau\left(\frac{x-x_{o}}{V_{1}}\right)+\frac{e_{w}}{2} h_{1}+f_{1}\right) q\left(x, x_{d}\right) d x_{d} d x+ \\
& \int_{L}^{B} \int_{L}^{x}\left(\tau\left(\frac{x-x_{d}}{V_{2}}\right)+\frac{e_{w}}{2} h_{2}+f_{2}\right) q\left(x, x_{d}\right) d x_{d} d x+ \\
& \frac{2\left(\alpha_{1}+\beta_{1} k_{1}\right)}{V_{1} h_{1}} L+\frac{2\left(\alpha_{2}+\beta_{2} k_{2}\right)}{V_{2} h_{2}}(B-L)
\end{aligned}
$$

subject to

$$
\begin{aligned}
& h_{1} Q_{T r u}^{1}(x) \leq k_{1} \eta_{1}, \forall x \in(0, L], \\
& h_{2} Q_{T r u}^{2}(x) \leq k_{2} \eta_{2}, \quad \forall x \in(L, B],
\end{aligned}
$$

Following the same logic used in solving Model 1, (A-6) and (A-7) are binding. Vehicle capacities can be written as functions of headways, i.e., $k_{1}=h_{1} \max _{\{0<x \leq L\}}\left(Q_{T r u}^{1}(x)\right) / \eta_{1}$ and $k_{2}=h_{2} \max _{\{L<x \leq B\}}\left(Q_{T r u}^{2}(x)\right) / \eta_{2}$, where $\max _{\{0<x \leq L\}}\left(Q_{T r u}^{1}(x)\right)$ is the highest through volume for the rail line and $\max _{\{L<x \leq B\}}\left(Q_{T r u}^{2}(x)\right)$ is the highest through volume for the bus route. Then objective (A-4) becomes a function of headways only. One can set the first-order partial derivatives of (A-4) with respect to $h_{1}$ and $h_{2}$ to be zero and find the optimal headways.

\section{Appendix 2 - Analysis with Demand Elasticity}

Model 1 in subsection 3.3 can be extended to consider elastic demand.

The new demand density function $\tilde{q}(x)$ is a linear function of the generalized user cost $\tilde{U}(x)$, as follows:

$$
\tilde{q}(x)=\left(\theta_{1}-\theta_{2} x\right)(1-\rho \tilde{U}(x))
$$


The positive coefficient $\rho$ measures how quickly demand drops with user cost. When $\tilde{U}(x)=\frac{1}{\rho}$, nobody travels from location $x \in(0, B]$.

When $0<x \leq \mathrm{L}, \tilde{U}_{1}(x)=g_{1}+\tau \frac{x}{V_{1}}+\frac{e_{w}}{2} h_{1}+f_{1}$, where $g_{1}$ is the rail fare; when $L<x \leq B$, $\tilde{U}_{2}(x)=g_{1}+g_{2}+\tau\left(\frac{x-L}{V_{2}}+\frac{L}{V_{1}}\right)+\frac{e_{w}}{2} h_{1}+\frac{e_{w}}{2} h_{2}+f_{2}$, where $g_{2}$ is the bus fare. Other symbols have been defined in the text, e.g., in Table 1.

The total revenue is expressed as:

$$
R=g_{1} \int_{0}^{B} \tilde{q}(x) d x+g_{2} \int_{L}^{B} \tilde{q}(x) d x
$$

The operator cost is the same as defined in Eq. (4).

The total user surplus of rail passengers is:

$$
\varphi=\int_{0}^{L} \int_{\tilde{U}_{1}(x)}^{\frac{1}{\rho}}\left(\theta_{1}-\theta_{2} x\right)\left(1-\rho u_{1}\right) d u_{1}+\int_{L}^{B} \int_{\tilde{U}_{2}(x)}^{\frac{1}{\rho}}\left(\theta_{1}-\theta_{2} x\right)\left(1-\rho u_{2}\right) d u_{2}
$$

Instead of minimizing the total cost under the fixed demand assumption, the social welfare, i.e., profit plus user surplus, is maximized when the demand function is elastic.

$$
\begin{aligned}
& \min _{\left\{g_{1}, g_{2}, h_{1}, h_{2}, k_{1}, k_{2}>0\right\}} g_{1} \int_{0}^{B} \tilde{q}(x) d x+g_{2} \int_{L}^{B} \tilde{q}(x) d x-\frac{2\left(\alpha_{1}+\beta_{1} k_{1}\right)}{V_{1} h_{1}} L-\frac{2\left(\alpha_{2}+\beta_{2} k_{2}\right)}{V_{2} h_{2}}(B-L) \\
&+\int_{0}^{L} \int_{\tilde{U}_{1}(x)}^{\frac{1}{\rho}}\left(\theta_{1}-\theta_{2} x\right)\left(1-\rho u_{1}\right) d u_{1}+\int_{L}^{B} \int_{\tilde{U}_{2}(x)}^{\frac{1}{\rho}}\left(\theta_{1}-\theta_{2} x\right)\left(1-\rho u_{2}\right) d u_{2}
\end{aligned}
$$

subject to

$$
\begin{aligned}
& h_{1} \int_{0}^{B} \tilde{q}(x) d x \leq k_{1} \eta_{1}, \\
& h_{2} \int_{L}^{B} \tilde{q}(x) d x \leq k_{2} \eta_{2},
\end{aligned}
$$




\section{References}

Casello, J. M., Lewis, G. M., Yeung, K., \& Santiago-Rodríguez, D. (2014). A Transit Technology Selection Model. Journal of Public Transportation, 17(4), 50-75.

Chang, S. K., \& Schonfeld, P. M. (1991a). Optimization models for comparing conventional and subscription bus feeder services. Transportation Science, 25(4), 281-298.

Chang, S. K., \& Schonfeld, P. M. (1991b). Integration of fixed-and flexible-route bus systems. Transportation Research Record, No. 1308, 51-57.

Chen, Y. J., Li, Z. C., \& Lam, W. H. (2015). Modeling transit technology selection in a linear transportation corridor. Journal of Advanced Transportation, 49(1), 48-72.

Cheng, W. C., \& Schonfeld, P. (2015). A Method for Optimizing the Phased Development of Rail Transit Lines. Urban Rail Transit, 1-11.

Chien, S., \& Schonfeld, P. (1998). Joint optimization of a rail transit line and its feeder bus system. Journal of Advanced Transportation, 32(3), 253-284.

Jara-Díaz, S. R., \& Gschwender, A. (2003). From the single line model to the spatial structure of transit services: corridors or direct?. Journal of Transport Economics and Policy (JTEP), $37(2), 261-277$.

Jara-Díaz, S. R., Gschwender, A., \& Ortega, M. (2012). Is public transport based on transfers optimal? A theoretical investigation. Transportation Research Part B: Methodological, 46(7), 808-816.

Kim, M. E., \& Schonfeld, P. (2013). Integrating bus services with mixed fleets.

Transportation Research Part B: Methodological, 55, 227-244.

Kim, M. E., \& Schonfeld, P. (2014). Integration of conventional and flexible bus services with timed transfers. Transportation Research Part B: Methodological, 68, 76-97.

Kim, M. E., \& Schonfeld, P. (2015). Maximizing net benefits for conventional and flexible bus services. Transportation Research Part A: Policy and Practice, 80, 116-133.

Li, Z. C., Lam, W. H., \& Wong, S. C. (2009). Optimization of a bus and rail transit system with feeder bus services under different market regimes. In Transportation and Traffic Theory 2009: Golden Jubilee (pp. 495-516). Springer US.

Li, Z. C., Lam, W. H., Wong, S. C., \& Sumalee, A. (2012). Design of a rail transit line for profit maximization in a linear transportation corridor. Transportation Research Part E: Logistics and Transportation Review, 48(1), 50-70.

Li, Z. C., Guo, Q. W., Lam, W. H., \& Wong, S. C. (2015). Transit technology investment and 
selection under urban population volatility: A real option perspective. Transportation Research Part B: Methodological, 78, 318-340.

Osuna, E. E., \& Newell, G. F. (1972). Control strategies for an idealized public transportation system. Transportation Science, 6(1), 52-72.

Parajuli, P. M., \& Wirasinghe, S. C. (2001). A line haul transit technology selection model. Transportation Planning and Technology, 24(4), 271-308.

Sivakumaran, K., Li, Y., Cassidy, M., \& Madanat, S. (2014). Access and the choice of transit technology. Transportation Research Part A: Policy and Practice, 59, 204-221.

Sivakumaran, K., Li, Y., Cassidy, M. J., \& Madanat, S. (2012). Cost-saving properties of schedule coordination in a simple trunk-and-feeder transit system. Transportation Research Part A: Policy and Practice, 46(1), 131-139.

Stutsman, J. (2002). Bus Rapid Transit or Light Rail Transit-How to Decide?: Los Angeles Case Study. Transportation Research Record, No. 1793, 55-61.

Sun, Y., \& Schonfeld, P. (2015a). Stochastic capacity expansion models for airport facilities. Transportation Research Part B: Methodological, 80, 1-18.

Sun. Y., and Schonfeld, P.M., (2015b). Schedule-Based Rail Transit Path Choice Estimation using Automatic Fare Collection Data. ASCE - Journal of Transportation Engineering, 142(1), 04015037.

Ting, C. J., \& Schonfeld, P. (2005). Schedule coordination in a multiple hub transit network. Journal of Urban Planning and Development, 131(2), 112-124.

Tirachini, A., Hensher, D. A., \& Jara-Díaz, S. R. (2010). Comparing operator and users costs of light rail, heavy rail and bus rapid transit over a radial public transport network. Research in Transportation Economics, 29(1), 231-242.

Van Mieghem, J. A. (2003). Commissioned paper: Capacity management, investment, and hedging: Review and recent developments. Manufacturing \& Service Operations Management, 5(4), 269-302.

Vuchic, V. R. (1976). Comparative analysis and selection of transit modes. Transportation Research Record, No. 559, 51-62. 